\title{
Gravitational and mass distribution effects on stationary superwinds.
}

\author{
G.A. Añorve-Zeferino* \\ École Polytechnique, Route de Saclay, 91128 Palaiseau, France
}

Accepted date. Received date; in original form date

\begin{abstract}
Here, we model the effect of non-uniform dynamical mass distributions and their associated gravitational fields on the stationary galactic superwind solution. We do this by considering an analogue injection of mass and energy from stellar winds and SNe. We consider both compact dark-matter and baryonic haloes that does not extend further from the galaxies optical radii $R_{\mathrm{opt}}$ as well as extended gravitationally-interacting ones. We consider halo profiles that emulate the results of recent cosmological simulations and coincide also with observational estimations from galaxy surveys. This allows to compare the analytical superwind solution with outflows from different kinds of galaxies. We give analytical formulae that establish when an outflow is possible and also characterize distinct flow regimes and enrichment scenarios. We also constraint the parameter space by giving approximate limits above which gravitation, self-gravitation and radiative cooling can inhibit the stationary flow. We obtain analytical expressions for the free superwind hydrodynamical profiles. We find that the existence or inhibition of the superwind solution highly depends on the steepness and concentration of the dynamical mass and the mass and energy injection rates. We compare our results with observational data and a recent numerical work. We put our results in the context of the mass-metallicity relationship to discuss observational evidence related to the selective loss of metals from the least massive galaxies and also discuss the case of massive galaxies.
\end{abstract}

Key words: hydrodynamics, gravitation, galaxies: starburst, ISM: jets and outflows

\section{INTRODUCTION}

Powerful outflows of gas are an ubiquitous feature of starforming galaxies at both low and high redshift. Early optical emission-lines surveys of nearby starburst galaxies carried out by Lehnert \& Heckman $(1995,1996)$ showed that several of the indicators that disclose the presence of a superwind, like extended line emission, shock-like emission-line ratios and broad emission lines, were positively correlated with infrared activity. Nowadays, the detection of superwinds in nearby galaxies through metal absorption-lines measurements, specially of the Na I D doublet $(\lambda \lambda 5890,5896)$, is an extended practice (Heckman et al. 2000; Martin 2005; Rupke \& Veilleux 2005; Rupke, Veilleux \& Sanders 2005a; Martin \& Bouché 2009; and references therein). Current studies of blue-shifted absorption lines have confirmed that many nearby infrared luminous $\left(L_{\mathrm{IR}} / L_{\odot}<10^{12}\right)$ and ultraluminous (ULIRGs, $L_{\mathrm{IR}} / L_{\odot}>10^{12}$ ) star-forming galaxies produce massive superwinds with velocities of several hun-

^ E-mail: gabriel-alejandro.anorve-zeferino@polytechnique.edu dreds of $\mathrm{km} \mathrm{s}^{-1}$. It has been also validated that the velocity, mass, momentum and energy of these outflows scale with the galaxy SFR, luminosity and mass (Rupke, Veilleux \& Sanders 2005b); fact that seems consistent with the picture of a pressure driven superwind (Chevalier \& Clegg 1985, CC85 hereafter). On the other hand, the bulk properties of the hot X-ray emitting haloes $\left(T \sim 10^{6}-10^{7} \mathrm{~K}\right)$ detected around some galaxies (Dahlen, Weaver \& Heckman 1998; Heckman et al. 2000; Strickland \& Heckman 2009) also agree with the predictions of simple superwind models (see Stevens \& Hartwell 2003).

The spectroscopical evidence of galactic scale outflows at intermediate and high redshifts is also ample. Recent infrared and radio studies point out to luminous and ultraluminous infrared galaxies at $z \geq 1$, as the hosts of the most intense star formation in the universe (Pérez-González et al. 2005; Chapman et al. 2005). At redshifts $z=2-3$, Lymanbreak galaxies (LBGs) are probably the most notable representatives of such extreme behaviour (see Heckman 2001 and references therein). The optical and infrared spectra of the LBG population at $z \sim 3$ present metal absorption lines 
and Ly $\alpha$ emission lines that are respectively blue-shifted and redshifted by hundreds of $\mathrm{km} \mathrm{s}^{-1}$ with respect to the galaxies rest frames (Pettini et al. 2001). The same has been observed in optical spectra of lensed Ly $\alpha$ emitting galaxies at $z>4$ (Frye, Broadhurst \& Benitez 2002). The redshifted Ly $\alpha$ signature has been also detected in LBGs at $z>5$ (e.g. Dawson, Spinrad, Stern et al. 2002; Tapken et al. 2007). As it has been pointed out by Heckman (2001) and Dawson et al. (2002), these observations are also consistent with the picture of an optically thick superwind expanding around the star-forming regions.

There is now a consensus on that galactic outflows could have a profound impact on the chemical evolution of galaxies and the dynamics of the IGM. They are expected to terminate star formation in some galaxies and to deposit heavy elements, heat and locally accelerate the IGM (Nath \& Trentham 1997; Benson \& Madau 2003).

Furthermore, superwinds seem to be one of the main agents $^{1}$ in the establishment of the observed strong correlation between galaxy mass and metallicity. A substantial amount of observational evidence points to a selective loss of metals from the least-massive galaxies and a full retention of the same by the most massive ones (Garnett 2002, Tremonti et al. 2004, Lee et al. 2006). The studies are coincident in reporting a saturation of the $\mathrm{O} / \mathrm{H}$ abundance (used as a surrogate for metallicity) for the most massive galaxies and a power-law-like behaviour for the intermediate-mass and least-massive galaxies. A popular view is that galactic superwinds are to blame for removing metals from the relative shallower gravitational potential wells of the least-massive galaxies. The absorption-lines and X-ray studies of superwinds from nearby galaxies carried out by Heckman et al. (2000) clearly support this trend. However, outliers from this empirical relationship have already been found in the form of low-mass high-metallicity dwarf galaxies (Peeples, Pogge \& Stanek 2008) and massive low-metallicity early-type ones (Peeples, Pogge \& Stanek 2009).

From another standpoint, the low metallicity $(Z \sim$ $\left.0.02-0.5 \mathrm{Z}_{\odot}\right)$ and high gas content of many dwarf irregular galaxies with historials of ongoing or recently finished starburst activity indicate that they are late-type objects. The latter is particularly true for blue compact dwarf galaxies (BCDs). It has been suggested that these young objects might be the predecessors of the predominantly early-type, gas-poor and low-metallicity population of $\mathrm{dE}$ and $\mathrm{dSph}$ dwarf galaxies (e.g. Dellenbusch et al. 2008). The favourite theory to explain the gas depletion that such transition implies is again based on starburst driven superwinds (Larson 1974, Dekel \& Silk 1986, Finlator \& Davé 2008).

The problem of how galaxies retain only certain amount of metals according to their masses has been already addressed analytically. Lynden-Bell (1992) proposed a simple heuristic model in which the fraction of the starburstproduced metals that are retained by a galaxy is proportional to the depth of the galaxy potential well for galaxies with escape velocities less than the outflow effective terminal velocity, i.e. $v_{\mathrm{e}}<V_{\infty}$, and asymptotes to full retention for the most massive galaxies with large $v_{\mathrm{e}}$. This heuristic

1 Galaxy mergers and tidal effects in dense cluster environments are out of the scope of this work. approach has been successfully applied by Heckman et al. (2000) and Heckman (2001) to explain their observational results. Under the assumption of an isothermal gravitational potential, they proposed a scheme in which an asymptotic full retention of metals is achieved when $v_{\mathrm{e}} \gg V_{\infty}$ and partial retention is proportional to $v_{\mathrm{e}}^{2}$.

Nevertheless, a self-consistent and simple analytical superwind hydrodynamical model incorporating gravitational effects and from which more general conclusions could be reached is still lacking. CC85 presented the standard galactic superwind model considering just the adiabatic, pressure driven expansion of the hot plasma resulting from the thermalization of individual stellar winds and supernovae ejecta inside of the starburst volume. Their model applies to fast superwinds for which gravitational effects are weak. This may be the case for low-mass galaxies; however, for massive galaxies, the binding gravitational energy can be comparable to the energy budget provided by the thermalization of the gas injected in the central regions $\left(v_{\mathrm{e}} \sim V_{\infty} \sim 1000 \mathrm{~km}\right.$ $\mathrm{s}^{-1}$, see Wang 1995). Furthermore, observational studies indicate that in many cases, galactic starburst episodes are centrally concentrated (e.g. Marlowe et al. 1995; Taniguchi, Trentham \& Shioya 1998; Cairós et al. 2003; Dellenbusch et al. 2008); an effect that has been so far neglected in superwind analytical models.

Here, we present a simple stationary sphericallysymmetric hydrodynamical model that incorporates gravitational effects and takes into account a central concentration of the dynamical mass with analogue mass and energy injection rates, with the aim of addressing, within the natural limitations of our approach, the following issues: (i) How does the galactic gravitational field affect the superwind hydrodynamical profiles and their related observables? (ii) How does the concentration of the dynamical mass and the mass and energy injection rates affect the superwind behaviour? (iii) What is the actual value of the asymptotic terminal speed that will determine the impact of the superwind on its surroundings when gravitational fields are taken into account? (iv) Under what circumstances can the outflow be inhibited? (v) What are the possible enrichment scenarios and when do they occur? (vi) What are the implications for the mass-metallicity relationship? As in previous approaches, a compromise will be established between the two usual suspects of determining the gas fate, $v_{\mathrm{e}}$ and $V_{\infty}$ (see e.g. Sharma and Nath 2012); however, here it will be done on a purely hydrodynamical basis and covering the case of gravitational potentials that can reproduce asymptotically flat rotation curves. This latter fact has not been taken into account in a previous work by Wang (1995) who presented an analytical superwind model considering power-law and logarithmic gravitational potentials.

As it has been previously cautioned, one must distinguish between outflows localized in extent (just a few kpc around the star forming region) from those that may be able to completely escape from the galaxies and have an impact on the IGM (see Mac Low \& Ferrera 1999). Here we analyze the former case, since as it has been pointed out by Heckman (2001), the intrinsic observable manifestations of galactic superwind are produced by material still relatively deep within the gravitational potential of the galaxy dynamical mass.

An overview of the organisation of the Paper is given 
next. The superwind model is presented at the beginning of Section 2. It is introduced initially for galaxies with compact haloes which do not exceed the optical radius $R_{\text {opt }}$. This can approximate the case of the biggest brightest spirals for which dark matter only accounts $15 \%$ of the total mass (Persic, Salucci \& Stel, 1996) and also SCUBA sources (Silich et al. 2010). In Section 2.1, the boundary conditions needed for a supersonic outflow are obtained. We also derive an expression for the asymptotic terminal speed and provide limits for which gravitation can establish different flow regimes. The associated enrichment scenarios are described qualitatively. In Section 3, we obtain analytical superwind solutions for the case of compact haloes. In general the dark-matter within $R_{\text {opt }}$ can vary from $0 \%$ to $30 \%-$ $70 \%$ (Persic \& Salucci, 1997); so, in Section 4 we extend our analytical model to galaxies with extended haloes (i.e. haloes with an extension much larger than $R_{\text {opt }}$ ). An analytical profile for the haloes is specified in Section 5. There, we also give the corresponding limits for the retention and escape of the superwind from its host galaxy. In Section 6 we present the resulting hydrodynamical profiles considering superwinds ejected by different kinds of galaxies and compare with a previous numerical work that considers massive galaxies with uniformly distributed dynamical masses and injection rates (Silich et al. 2010). We contrast the predictions of our model with observational data in Section 7 . The conclusions are presented in Section 8. In Appendixes $\mathrm{A}$ and $\mathrm{B}$, formulae that establish the circumstances under which self-gravitation and radiative cooling can inhibit the superwind solution are given.

\section{THE SUPERWIND MODEL}

Let's define first the parameters and variables of the model. The superwind is powered by a central spheroidal object that represents either a galaxy or a protogalaxy. Each central object is defined by a set of three parameters and a normalised density profile $\left\{r_{\mathrm{sc}}, \dot{E}_{\text {eff }}, \dot{M}_{\text {eff }}, \rho_{\mathrm{s}}\right\}$ : a characteristic object radius, ${ }^{2} r_{\mathrm{sc}}$; the effective energy deposition rate, $\dot{E}_{\text {eff }}$; the effective mass deposition rate, $\dot{M}_{\text {eff }}$; and a normalized spatial distribution, $\rho_{\mathrm{s}}$. The latter is used to trace the densities of the dynamical mass (both the stellar and dark matter components) and the mass and energy deposition rates inside the galaxy, i.e. they are assumed to be proportional to $\rho_{\mathrm{s}}$. In reality, the deposition rates are proportional to the star formation rate, which in turn is related to the surface density of gas. So, considering a single $\rho_{\mathrm{s}}$ is a drawback in our model; however, this affects only the central region $\left(r<r_{s c}\right)$ but gives anyway coherent values of the hydrodynamical variables at $r_{\mathrm{sc}}$.

We have followed Strickland \& Heckman (2009) in assuming that $\dot{E}_{\text {eff }}$ and $\dot{M}_{\text {eff }}$ are given by

$\dot{E}_{\text {eff }}=\epsilon \zeta \dot{E}_{\mathrm{SN}+\mathrm{SW}}$

2 The characteristic radius of a galaxy bulge or nucleus, e.g. the optical radius $R_{\mathrm{opt}}$. and

$\dot{M}_{\mathrm{eff}}=\zeta \dot{M}_{\mathrm{SN}+\mathrm{SW}}+\dot{M}_{\mathrm{cold}}=\beta \zeta \dot{M}_{\mathrm{SN}+\mathrm{SW}}$,

where $\dot{E}_{\mathrm{SN}+\mathrm{SW}}$ and $\dot{M}_{\mathrm{SN}+\mathrm{SW}}$ are the energy and mass deposition rates due to stellar winds and $\mathrm{SNe}$ within the whole central volume, $\zeta \leq 1$ is a participation factor that removes negligible thermalization regions, and $\epsilon \leq 1$ is the mean thermalization efficiency. Similarly, $\beta \geq 1$ is a mass loading factor that accounts for the incorporation of ambient gas within the central volume. The effective terminal speed is then given by

$V_{\infty}=\left(\frac{2 \dot{E}_{\mathrm{eff}}}{\dot{M}_{\mathrm{eff}}}\right)^{1 / 2}=\left(\frac{2 \epsilon \dot{E}_{\mathrm{SN}+\mathrm{SW}}}{\beta \dot{M}_{\mathrm{SN}+\mathrm{SW}}}\right)^{1 / 2}=$

$12.6\left(\frac{2 \epsilon \dot{E}_{38}}{\beta \dot{M}_{\odot}}\right)^{1 / 2} \mathrm{~km} \mathrm{~s}^{-1}$.

where $\dot{E}_{38}$ is the energy deposition rate in units of $10^{38} \mathrm{erg}$ $\mathrm{s}^{-1}$ and $\dot{M}_{\odot}$ is the mass deposition rate in $M_{\odot} \mathrm{yr}^{-1}$. The mass, momentum and energy conservation laws for the flow within $r \leq r_{\mathrm{sc}}$ are

$\frac{1}{r^{2}} \frac{\mathrm{d}\left(\rho u r^{2}\right)}{\mathrm{d} r}=\dot{q}_{\mathrm{m}}$

$\rho u \frac{\mathrm{d} u}{\mathrm{~d} r}=-\frac{\mathrm{d} P}{\mathrm{~d} r}-\dot{q}_{\mathrm{m}} u-\rho \nabla \phi$

and

$\frac{1}{r^{2}} \frac{\mathrm{d}\left[\rho u r^{2}\left(\frac{1}{2} u^{2}+(\eta+1) \frac{P}{\rho}\right)\right]}{\mathrm{d} r}=\dot{q}_{\mathrm{e}}-\rho u \nabla \phi$.

Here, $r$ is the radial coordinate, $u$ is the velocity, $P$ is the pressure, $\rho$ is the gas density and $\dot{q}_{\mathrm{m}}$ and $\dot{q}_{\mathrm{e}}$ are the mass and energy deposition rates per unit volume, respectively. Here both are proportional to $\rho_{\mathrm{s}}$. We also have assumed an ideal polytropic flow with polytropic index $\eta$. The case $\eta=3 / 2$ is equivalent to the $\gamma=5 / 3$ case and $c^{2}=(\eta+1) P / \eta \rho$ is equivalent to the squared sound speed (Añorve-Zeferino, Tenorio-Tagle \& Silich 2009). The gravitational acceleration is $-\nabla \phi=-G M(r) / r^{2}$, where $\mathrm{M}(\mathrm{r})$ is the cumulative dynamical mass (see Section 2.1) and $G$ the constant of universal gravitation.

As it was mentioned in the introduction we concentrate first on the case of compact haloes. So, The conservation laws that govern the flow outside of the central volume $\left(r>r_{\mathrm{sc}}\right)$ are

$\frac{1}{r^{2}} \frac{\mathrm{d}\left(\rho u r^{2}\right)}{\mathrm{d} r}=0$

$\rho u \frac{\mathrm{d} u}{\mathrm{~d} r}=-\frac{\mathrm{d} P}{\mathrm{~d} r}-\rho \nabla \phi$

and

$\frac{1}{r^{2}} \frac{\mathrm{d}\left[\rho u r^{2}\left(\frac{1}{2} u^{2}+(\eta+1) \frac{P}{\rho}\right)\right]}{\mathrm{d} r}=-\rho u \nabla \phi$. 
In the last equation, $-\nabla \phi=-G M_{\mathrm{DM}} / r^{2}$ and $M_{\mathrm{DM}}$ is the total dynamical mass of the central object. We neglect the effect of cooling and self-gravitation. However, we evaluate their impact in Appendixes A and B providing a justification for this assumption. Here, we adopt a symmetrypreserving energy balance approach - similar to that present in the CC85 model and in the 3D simulations of Recchi, Matteucci \& D'Ercole (2001) - that will lead us to relations for comparing the relative strengths of the thermalization and the gravitational potential in a similar manner than in the heuristic proposal of Lynden-Bell, but with a patent hydrodynamical basis. None the less, in order to compare such strengths, we consider physically motivated mass distributions that can recover flat rotation curves by choosing $\rho_{\mathrm{s}}$ in a suitable manner.

We incorporate in our model the effect of the gravitational field and obtain an analytical solution for the external zone $\left(r>r_{\mathrm{sc}}\right)$. For completeness, we obtain numerically the hydrodynamical profiles corresponding to the inner region $\left(r \leq r_{\mathrm{sc}}\right)$. Nevertheless, we use the analytic integrated forms of equations (4) and (6) to obtain the proper boundary conditions at $r_{\mathrm{sc}}$ and unveil some relevant physics, as shown below.

\subsection{Boundary conditions and the existence of the superwind solution}

We impose boundary conditions that warrant the continuity of the fluxes across the central object surface. The boundary condition for the mass flux, $F_{\mathrm{m}}$, is

$F_{\mathrm{m}}\left(r_{\mathrm{sc}}\right)=\frac{\dot{M}_{\mathrm{eff}}}{4 \pi}$.

Although trivial, this relation ensures consistency with the stationary mass flux associated to a localised mass injection.

We will focus on superwinds that expand transonically outside of the central object characteristic radius. Such velocity profiles are only possible if the flow attains a Mach number equal to unity at $r_{\mathrm{sc}}$, i.e. if $u_{\mathrm{sc}}=c_{\mathrm{sc}}$. In order to establish the adequate boundary condition for the energy flux, we need to define first $\rho_{s}$. We consider two kinds of normalized spatial distributions: a truncated version of a profile introduced by Dehnen (1993) and a uniform distribution (the almost always casted out assumption; see, however, Ji, Wang \& Kwan 2006). Their respective expressions are

$\rho_{s}=\frac{(3-\alpha)}{4 \pi}\left(\frac{r_{\mathrm{sc}}+a}{r_{\mathrm{sc}}}\right)^{3-\alpha} \frac{a}{r^{\alpha}(r+a)^{4-\alpha}}$

and

$\rho_{s}=\frac{3}{4 \pi R_{\mathrm{sc}}^{3}}$.

A huge advantage of the Dehnen-like distribution is that it depends on a steepness parameter ${ }^{3} \alpha \in[0,5 / 2)$ and a scale parameter $a \in(0, \infty)$. These parameters determine the internal structure of the central object, a feature often

3 For reasons related to the convergence of the energy integral for a spherical stationary flow, we further constraint the original interval defined by Dehnen: $\alpha \in[0,3)$. ignored in analytical superwind models, but that is decisive in determining the fate of the injected gas. For $\alpha=0,1,2$ we obtain normalized truncated versions of a plateau-like and the Hernquist (1990) and Jaffe (1983) profiles, respectively. Written in terms of the normalized radius $R=r / r_{\mathrm{sc}}$ and the concentration parameter $A=a / r_{\mathrm{sc}}$, the explicit expressions for the cumulative mass corresponding to the Dehnen-like profile and the uniform distribution are

$M(R)=M_{\mathrm{DM}}(1+A)^{3-\alpha}\left(\frac{R}{R+A}\right)^{3-\alpha}$

$M(R)=M_{\mathrm{DM}} R^{3}$,

respectively: Equivalent expressions could be written for $\dot{E}(R)$ and $\dot{M}(R)$ by replacing $M_{\mathrm{DM}}$ by $\dot{E}_{\text {eff }}$ and $\dot{M}_{\text {eff }}$. In Fig. 1 , we present the normalized cumulative dynamical mass for the Jaffe, Hernquist and plateau-like profiles using a small concentration parameter and a large one. For small values of $A$, most of the mass is contained in the innermost regions of the central object, although at different degrees. For very large values of $A$, the cumulative mass varies almost linearly with $R$ for the Jaffe-like profile; it varies as $\sim R^{2}$ for the Hernquist-like one and (almost) reproduces the behaviour resulting from the uniform distribution for the plateau-like profile, i.e. it varies as $\sim R^{3}$. For the latter two cases, most of the mass is contained in the outer layers of the (proto) galaxy. ${ }^{4}$ The profiles scale accordingly for other values of $\alpha$ and $A$. The circular velocity profiles, $v_{\text {rot }}=\sqrt{G M(r) / r}$, corresponding to the distributions showed in Fig. 1 are displayed normalized to $\sqrt{G M_{\mathrm{DM}}}$ in Fig. 2. The escape velocity is given by $v_{\mathrm{e}}(r)=\sqrt{2} v_{\text {rot }}$. One can recover asymptotically quasi-flat rotation curves for truncated Dehnen profiles with $\alpha \leq 1$ and small values of $A$. Thus, we can study the effect of the distribution of the dynamical mass and the energy and mass deposition rates per unit volume in a more general setting than previous works.

With equations (11) and (12), one can explicitly define $\dot{q}_{\mathrm{e}}=\dot{E}_{\mathrm{eff}} \rho_{\mathrm{s}}, \dot{q}_{\mathrm{m}}=\dot{M}_{\mathrm{eff}} \rho_{\mathrm{s}}$ and $M=4 \pi M_{\mathrm{DM}} \int_{0}^{r} \rho_{s} r^{\prime 2} d r^{\prime}$ and integrate equations (4) and (6) to obtain a Bernoullilike equation (see Añorve-Zeferino et al. 2009). From the integration of (4), equation (10) follows immediately. The integration of (6) yields

$\frac{1}{2} u^{2}+(\eta+1) \frac{P}{\rho}=$

$\frac{1}{2} V_{\infty}^{2}-\frac{1}{(5-2 \alpha)}\left(\frac{r_{\mathrm{sc}}+a}{r_{\mathrm{sc}}}\right)^{3-\alpha}\left(\frac{r}{r+a}\right)^{2-\alpha} \frac{G M_{\mathrm{DM}}}{a}$

and

$\frac{1}{2} u^{2}+(\eta+1) \frac{P}{\rho}=\frac{1}{2} V_{\infty}^{2}-\frac{1}{5} \frac{G M_{\mathrm{DM}} r^{2}}{r_{\mathrm{sc}}^{3}}$,

for the Dehnen and the uniform distribution, respectively. These equations will determine the qualitative character of

4 Notice that the behaviour for large $A$ is different from that of the original Dehnen profiles. This is due to the truncation, since now and respectively, $\rho_{\mathrm{S}} \sim r^{-2} \rho_{\mathrm{S}} \sim r^{-1}$ and $\rho_{s} \sim$ constant in the whole volume as $a \rightarrow \infty$. 


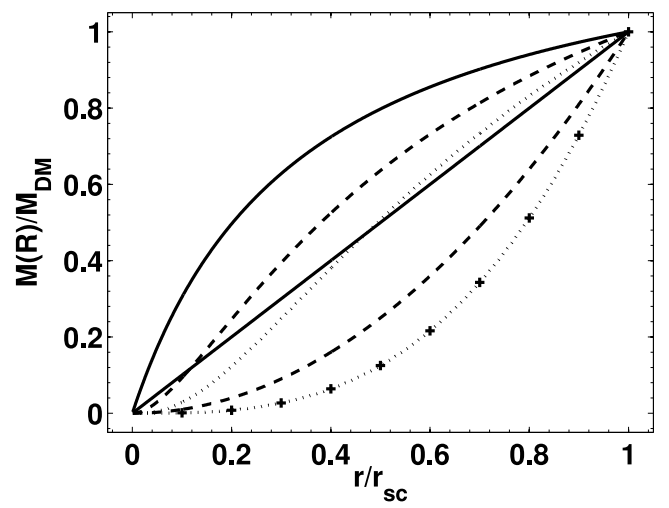

Figure 1. Normalized cumulative mass for different $\rho_{\mathrm{s}}$. The solid, dashed and dotted lines correspond to Jaffe $(\alpha=2)$, Hernquist $(\alpha=1)$ and plateau-like $(\alpha=0)$ truncated profiles, respectively. The concentration parameters are $A=0.34$ (upper lines) and $A=1000$ (lower lines). The crosses correspond to a uniform mass distribution.

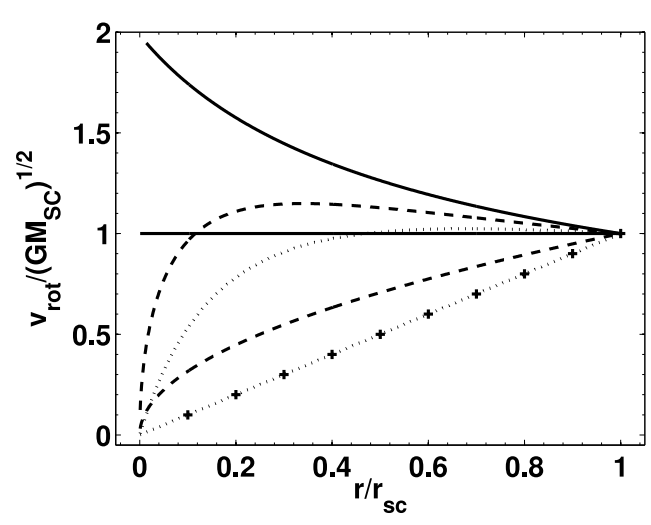

Figure 2. Rotation speed normalized to $\left(G M_{\mathrm{DM}}\right)^{1 / 2}$ for the profiles showed in Fig. 1. Again, the upper lines correspond to $A=0.34$ and the lower lines to $A=1000$.

the solution. For instance, note that for the Jaffe-like profile $(\alpha=2)$, the sum of the kinetic energy and the enthalpy per unit mass is reduced everywhere within the central volume by the same amount (i.e. by a constant) due to the steepness of the associated gravitational potential, whereas for the uniform distribution the reduction is proportional to $r^{2}$.

For convenience and future use we define the dimensionless variable $V_{\mathrm{e}}$ as the squared escape velocity at $r_{\mathrm{sc}}$ to the squared effective terminal speed:

$V_{\mathrm{e}}=\frac{v_{\mathrm{e}}^{2}}{V_{\infty}^{2}}=\frac{2 G M_{\mathrm{DM}}}{r_{s c} V_{\infty}^{2}}$.

From the RHSs of equations (15) and (16) one can determine whether the inner stationary solution exists or not. There is no solution at all when

$V_{\mathrm{e}} \geq V_{\mathrm{e}, \mathrm{cb}}=\frac{(5-2 \alpha)}{1+\frac{1}{A}}$ and $V_{\mathrm{e}} \geq V_{\mathrm{e}, \mathrm{cb}}=5$,

respectively. The resulting threshold lines for $V_{\mathrm{e}}$ are shown in Fig. 3. They are obtained by taking the equality signs in the equations above; so, according to the case, no solution exists above the respective line. For the Dehnen-like profiles this depends on the numerical values of $\alpha$ and $A$.

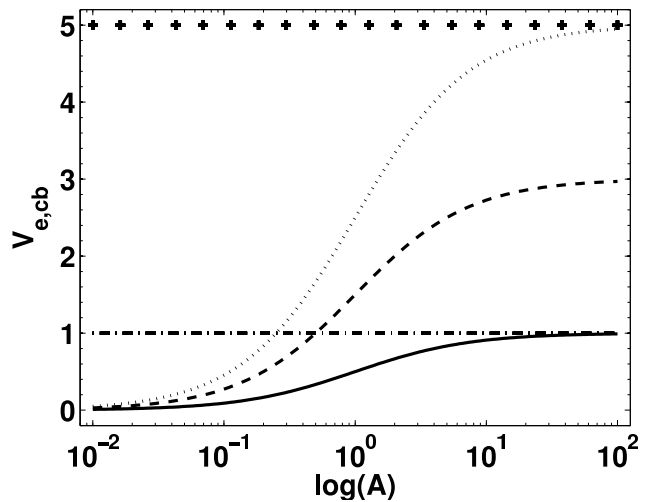

Figure 3. Threshold lines for the normalized squared escape velocity above which the gas cannot leave the central object, $V_{\mathrm{e}, \mathrm{cb}}$, as a function of the concentration parameter. The lines correspond to the same kind of profiles showed in Fig. 1. The dash-dotted line is included as a reference.

Since $v_{\mathrm{e}}^{2} \propto M_{\mathrm{DM}} / r_{\mathrm{sc}}$, the more massive and more compact the object, the closer it will be to the respective threshold line and the more difficult will be for the injected gas to escape from it, as expected. On the other hand, $V_{\infty}^{2}$ is directly proportional to $\dot{E}_{\text {eff }}$ and inversely proportional to $\dot{M}_{\text {eff }}$. Hence, poor energy injection rates (low $\dot{E}_{\mathrm{SN}+\mathrm{SW}}$ ), inefficient thermalization $(\epsilon \ll 1)$, high mass injections (large $\left.\dot{M}_{\mathrm{SN}+\mathrm{SW}}\right)$ and mass loading $(\beta \gtrsim 1)$ can contribute too to maintaining the gas bound, as it is also expected. However, there are two characteristics of the inequalities in (18) that are not so obvious. First, for the uniform distribution the gas can escape from the central object even when $V_{\mathrm{e}}>1$. The same occur for models with $\alpha<2$ and intermediate to large values of $A$. Second, and as it can be seen in Fig. 3, the gas can remain bound even when $V_{\mathrm{e}} \ll 1$ provided that $A$ remains sufficiently small. For models with $\alpha>2$ the later can occur for large values of the concentration parameter $A$.

The first effect occurs because, as explained before, for the uniform distribution and the Dehnen profiles with $\alpha<2$ and large $A$, most of the mass and energy are injected in the outer regions of the (proto-) galaxy, where most of the binding mass (both stellar and dark matter) is also located. A parcel of gas at a position $r$ within this external zone is driven out by the high pressure gradient more effectively than it is attracted by the gravitational force exerted by the relatively voider central regions (where less mass is concentrated), and thus, the gas has enough time to cross the object boundary before being pulled back by the gravitational field, although it can be with a rather slow velocity. The second effect, present for the Dehnen-like profiles with relatively small $A$ (this depends on the value of $\alpha$ ), can be explained in analogue terms: in this case, the central gravitational potential is so dominant that even for small $V_{\mathrm{e}}$, the injected gas adopts a steep density profile, and as a result, the energy per unit mass at $r_{\mathrm{sc}}$-in particular the enthalpyis small. In consequence, the gas cannot escape from the central object. All this implies that only galaxies with their parameter $V_{\mathrm{e}}$ above the threshold defined by (18) would have deep enough potential wells to keep their injected gas at $r<r_{\mathrm{sc}}$ and enrich themselves with their processed metals in a total closed-box scenario.

We will proceed to give in advance two results coming 


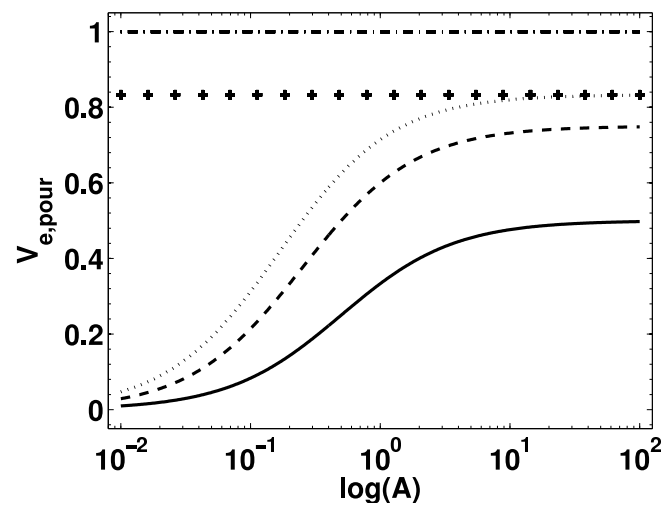

Figure 4. Threshold lines for the normalized squared escape velocity below which the flow is fully stationary. The lines correspond to the same kind of profiles than in Fig. 3.

from the analytic solution for the external zone, Section 3 , equations (28) and (29). The first one is that stationary flows that escape the central region with $V_{\mathrm{e}}$ above certain threshold value will have their stationary solution inhibited in the outer zone, $r>r_{\mathrm{sc}}$. If the stationary central wind has enough ram pressure, it will be difficult for the outer non-stationary flow to cross again the central object boundary, and thus, most likely, a complex morphology, filamentary and/or turbulent (stirred up by galactic rotation), will result in the outer zone from the interaction of both flows ('outpouring'). This would be the case when $V_{\mathrm{e}}$ is equal or somewhat less than 1 . However, the flow can also be eventually reinserted into the inner zone (Silich et al. 2010) and rain down over the central object $\left(1<V_{\mathrm{e}}<V_{\mathrm{e}, \mathrm{cb}}\right.$, 'inpouring'). The threshold lines that separate flows in these 'outpouring' (outflow) and 'inpouring' (inflow/rain back) regimes from flows in a fully stationary regime are given by

$V_{\mathrm{e}} \geq V_{\mathrm{e}, \mathrm{pour}}=\frac{5-2 \alpha}{6-2 \alpha+\frac{1}{A}}$ and $V_{\mathrm{e}} \geq V_{\mathrm{e}, \mathrm{pour}}=\frac{5}{6}$.

The threshold lines for the cases $\alpha=0,1,2$ and the uniform distribution are presented in Fig. 4. They clearly reflect that for a fully stationary outflow $V_{\mathrm{e}}<1$, i.e. the parameters of the flow need to be such that their combination falls below the respective threshold line.

The second result is that not all fully-stationary superwinds behave in the same way. Some will have bounded (in the mathematical sense) accelerating solutions and some will have bounded decelerating ones. Using equations (28) and (29), we find that for a fully-stationary flow, gravity fixes the asymptotic terminal speed to ${ }^{5}$

$V_{\mathrm{g}}=\left(1-\frac{6-2 \alpha+\frac{1}{A}}{5-2 \alpha} V_{\mathrm{e}}\right)^{1 / 2} V_{\infty}$

From the relation between the velocity and the sound

5 i.e. the true value of the superwind speed far away from the object, which is clearly distinct from $V_{\infty}$ defined by equation (3). Hereafter, we will give expressions only associated to the truncated Dehnen profiles. The equivalent ones for the uniform distribution can be obtained from them by taking $\alpha=0$ and $A \rightarrow \infty$.

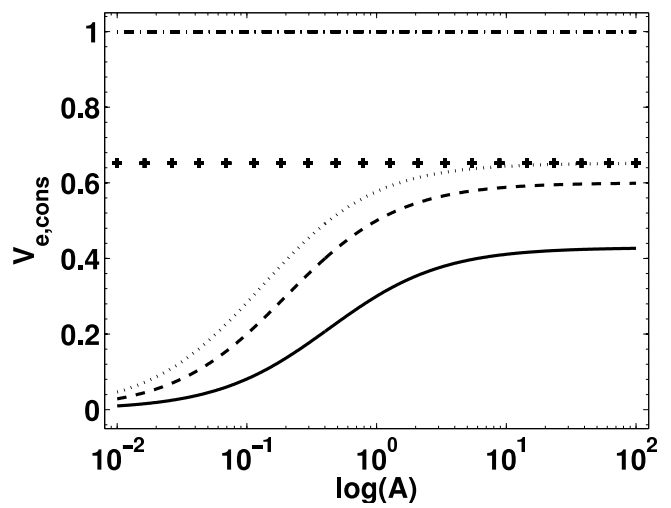

Figure 5. Threshold lines for the normalized squared escape velocity below which the flow has accelerating stationary solutions. The lines correspond to the same kind of profiles than in Fig. 3 . The outflows with decelerating supersonic solutions are compressed between these lines and those shown in Fig. 4.

speed at $r_{\mathrm{sc}}, u_{\mathrm{sc}}=c_{\mathrm{sc}}$, it follows that a fully stationary free superwind cannot have an accelerating velocity profile if

$V_{\mathrm{e}} \geq V_{\mathrm{e}, \mathrm{cons}}=\frac{2 \eta(5-2 \alpha)}{(2 \eta+1)(5-2 \alpha)+2 \eta\left(1+\frac{1}{A}\right)}$.

In this formula, the equality sign corresponds to an almost constant external velocity profile with $V_{\mathrm{g}}=u_{\mathrm{sc}}$. The corresponding threshold lines are shown in Fig. 5, where we have assumed that $\eta=3 / 2$. This assumption will be used in all successive quantitative calculations.

When the equality in equation (21) is satisfied, there is such a concert between gravity and thermalization that the asymptotic terminal speed depends only on the escape velocity (or rotation speed) at $r_{\mathrm{sc}}$ :

$V_{\mathrm{g}, \mathrm{th}}=(2 \eta)^{-1 / 2} v_{\mathrm{e}}=\eta^{-1 / 2} v_{\mathrm{rot}}$.

This is a very interesting result; specially, for the case of asymptotically flat rotation curves.

For the particular case $A \rightarrow \infty$ and $\alpha=0$, our analysis concurs with and can explain the numerical results obtained by Silich et al. (2010) for the case of uniformly distributed dynamical masses and injection rates. They associated the pouring regime with an in-falling bound wind, which they estimated to occur roughly when $c_{\mathrm{sc}}<v_{\mathrm{e}} / 2$. Such implicit estimation is consistent with our more exact limit for the regime. Additionally, they didn't give any limits for separating bounded accelerating from bounded decelerating fully stationary solutions, although they obtained both types of velocity profiles through their numerical calculations. Here, we have presented explicit upper and lower analytical limits for all possible regimes using more realistic distributions.

Although subtle, the difference between the lines showed in Fig. 4 and Fig. 5 is extremely important. Galaxies with their parameter $V_{\mathrm{e}}$ close to the lines showed in Fig. 4 will generate (in principle) fully stationary superwinds with very low asymptotic terminal speeds. Such a decrease of the velocity can lead to high densities and, in consequence, the flow could become both gravitationally and radiatively unstable and eventually enter into the outpouring or even the inpouring regime. Since this time, the gas could have been 
polluted by mixing with material external to the generating (proto-) galaxy before being reinserted, the inpouring (inflow) regime corresponds to an open-box metal enrichment scenario, or perhaps impoverishment or neither of both; see the general theorems presented by Edmunds (1990) and the work of Dalcanton (2007).

Additionally, one must consider that these limits are general upper bounds. As suggested above, effects like radiative cooling and self-gravitation can modify the flow. In Appendixes A and B we evaluate the effect of self-gravitation and also give an approximate analytical expression for the cooling threshold lines.

\section{ANALYTICAL SOLUTION FOR THE CASE OF COMPACT HALOES}

\subsection{The central superwind}

For the central regions $r<r_{\mathrm{sc}}$, we present a numerical solution to the conservation laws and limit our study to the case $\alpha \leq 1$. For such values of $\alpha$, the flow have finite central densities that can withstand self-gravity and catastrophic cooling effects (sections A and B) for a wide range of parameters. Thus, for the cases here analyzed, no bimodal-like behaviour will be obtained when the gravitational field allows a solution to exist.

Below, we give the values of the hydrodynamical variables at $r_{\mathrm{sc}}$ and the value of the central temperature, which can be useful to characterize the flow:

$u_{\mathrm{sc}}^{2}=\frac{V_{\infty}^{2}}{(2 \eta+1)}\left(1-\frac{\left(1+\frac{1}{A}\right)}{(5-2 \alpha)} V_{\mathrm{e}}\right)$

$\rho_{\mathrm{sc}}=\frac{\dot{M}_{\mathrm{eff}}}{4 \pi r_{\mathrm{sc}}^{2} u_{\mathrm{sc}}}$

$T_{\mathrm{sc}}=\frac{\eta \mu_{\mathrm{i}}}{(\eta+1) k} u_{\mathrm{sc}}^{2}$,

and

$T_{\mathrm{c}}=\frac{\mu_{\mathrm{i}}}{2(\eta+1) k} V_{\infty}^{2}$,

where $\mu_{\mathrm{i}}$ is the mean mass per particle for a ionized gas and $k$ the Boltzmann constant. We remark that at the threshold for an accelerating solution, $u_{\mathrm{sc}}=V_{\mathrm{g}}=\eta^{-1 / 2} v_{\text {rot }}$.

\subsection{The general solution for the free wind with compact haloes}

The effect of the gravitational field and the spatial distribution $\rho_{\mathrm{s}}$ on the free superwind hydrodynamical profiles will be characterized analytically. The integration of equations (7) and (9) and the application of the boundary conditions (10) and (15) yield explicit algebraic relations among the hydrodynamical variables

$\rho=\frac{\dot{M}_{\mathrm{eff}}}{4 \pi u r^{2}}$ and

$\frac{1}{2} u^{2}+(\eta+1) \frac{P}{\rho}=\frac{1}{2} V_{\mathrm{g}}^{2}-\phi$,

where $\phi=-G M_{\mathrm{DM}} / r$ and

$\frac{1}{2} V_{\mathrm{g}}^{2}=\frac{1}{2} V_{\infty}^{2}-\frac{G M_{\mathrm{DM}}}{r_{\mathrm{sc}}}-\frac{\left(1+\frac{1}{A}\right)}{(5-2 \alpha)} \frac{G M_{\mathrm{DM}}}{r_{\mathrm{sc}}}$.

Again, for convenience, we will work in terms of dimensionless variables. For the outer zone they are:

$R=\frac{r}{r_{\mathrm{sc}}} ; \quad U=\frac{u^{2}}{V_{\mathrm{g}}^{2}}$ and $\Phi=-\frac{V_{\mathrm{eg}}}{R}$,

where $V_{\text {eg }}$ is given by

$V_{\mathrm{eg}}=\frac{v_{\mathrm{e}}^{2}}{V_{\mathrm{g}}^{2}}=\frac{2 G M_{\mathrm{DM}}}{r_{s c} V_{\mathrm{g}}^{2}}$.

Notice that the normalization of the velocity related terms is now made to the value of the asymptotic gravitationallyestablished terminal speed. After combining equations (27)(28) with the equation of conservation of momentum, we arrive to the following differential equation

$U^{\prime}=\frac{[1-(U+\Phi)]}{(\eta+1)}\left(\frac{2}{R}+\frac{1}{2 U} U^{\prime}\right)+\frac{(U+\Phi)^{\prime}}{(\eta+1)}-\Phi^{\prime}$,

where the prime symbol indicates differentiation with respect to $R$.

Applying consecutively the changes of variable $\xi=U+$ $\Phi$ and $w=(2 \eta+1) \xi-2 \eta \Phi-1$ we arrive to an Abel differential equation in non-canonical form. Without loss of generality, let's assume initially that $\eta=3 / 2$, value that corresponds to a pseudo-adiabatic gas. Thus, we obtain

$R w w^{\prime}=-w^{2}+2 w+3\left(1+\frac{V_{\mathrm{eg}}}{R}\right)$.

We can reduce this equation to the canonical form by applying the change of variable $W=w R$. The resulting equation is

$W W^{\prime}=2 W+3 R\left(1+\frac{V_{\mathrm{eg}}}{R}\right)$.

We can further simplify the above equation if we work in terms of $x=R+V_{\mathrm{eg}}$ as the independent variable and $y=W / x$ as the dependent one. Proceeding this way, the differential equation transforms into a separable one

$y y^{\prime}=\frac{-y^{2}+2 y+3}{x}$.

This last equation is elementary and can be integrated using partial fractions. The solution is

$x=D_{0}(y+1)^{-1 / 4}(3-y)^{-3 / 4}$,

where $D_{0}$ is an integration constant. Returning to our original dimensionless variables, we have that

$R=D U^{-1 / 4}\left[1-U+\frac{V_{\mathrm{eg}}}{R}\right]^{-3 / 4}$, 
where $D$ is also a constant. By direct substitution, it is easy to show that the generalization

$R=D U^{-1 / 4}\left[1-U+\frac{V_{\mathrm{eg}}}{R}\right]^{-\eta / 2}$

is the general solution of equation (32) for arbitrary $\eta$. A similar result can be obtained for any conservative force term included in the RHS of the equation of energy. For our case, the constant $D$ is given by

$D=\left(\frac{1}{2 \eta+1}\right)^{1 / 4}\left[\frac{2 \eta\left(1+V_{\mathrm{eg}}\right)}{2 \eta+1}\right]^{\eta / 2}$.

In the absence of the gravitational field, $V_{\text {eg }}$ is identically zero, and thus we recover the CC85 superwind solution written in terms of the velocity (see Cantó, Raga \& Rodríguez 2000 and Añorve-Zeferino et al. 2009).

\subsection{Branches and parametric form of the general solution}

Certainly, the inclusion of the gravitational field and the departure from a uniform distribution will modify the topology of the outer flow. Nevertheless, we can use an analogue of equation (36) to gain some insight about the qualitative behaviour of the solution and, for the sake of accuracy and simplicity, obtain a parametric form of (38) that will avoid the need of using a numerical root finder (at least for important values of $\eta$ ). For general $\eta$, we have that

$x\left(x-V_{\text {eg }}\right)^{\frac{2 \eta-3}{4}}=D_{0}(y+1)^{-\frac{1}{2 \eta+1}}(2 \eta-y)^{-\frac{2 \eta}{2 \eta+1}}$

with

$x=R+V_{\mathrm{eg}}$,

$y=\left[\frac{(2 \eta+1) U}{1+\frac{V_{\mathrm{eg}}}{R}}-1\right]$,

and

$D_{0}=\left(1+V_{\text {eg }}\right)(2 \eta)^{\frac{2 \eta}{2 \eta+1}}$

In Fig. 6, we plot equation (40) normalized to the value of $D_{0}$ and taking the LHS as a function of $y$. The different branches of the solution are shown. The values of $y$ corresponding to expanding wind solutions are bounded to the interval $(-1,2 \eta)$. Equation (40) has a single global minimum at $y=0$ which corresponds to the sonic point.

Hence, The supersonic free wind corresponds to the branch $y \in[0,2 \eta)$. By taking $y$ as a parameter, we can obtain the hydrodynamical profiles as follows:

(i) Make $y$ vary between 0 and $2 \eta$ and then evaluate the RHS of equation (40). Then find the respective values of $x$. For $\eta=3 / 2$ this is straightforward.

(ii) Using equation (41), find the corresponding values of the normalized radius, $R=x-V_{\text {eg }}$.

(iii) With the values of $R$ and $y$, find $U$ from equation (42), $U=(y+1)\left(1+V_{\mathrm{eg}} / R\right) /(2 \eta+1)$. Then de-normalize to find the actual radius $\left(r=r_{\mathrm{sc}} R\right)$ and velocity $\left(u=V_{\mathrm{g}} U^{1 / 2}\right)$.

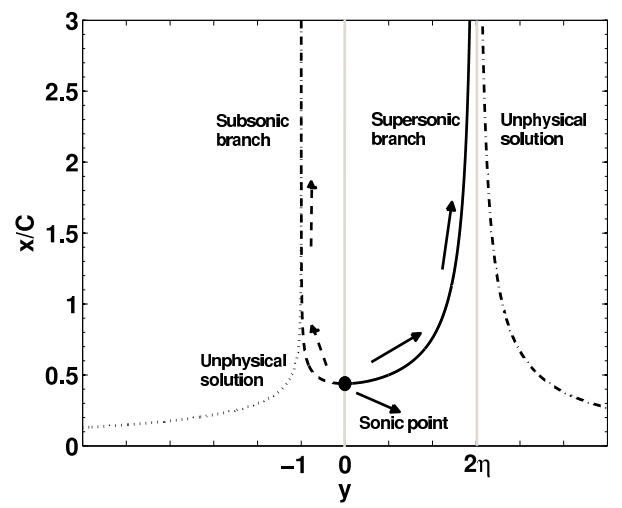

Figure 6. Branches of the general superwind solution for $r>r_{\mathrm{sc}}$. For $y \in(-1,0)$ we have a subsonic expansion. For $y \in[0,2 \eta]$, the expansion is supersonic.

(iv) From equation (28), the relation $c^{2}=(\eta+1) P / \eta \rho$ and the equation of state, $P=k \rho T / \mu_{\mathrm{i}}$, obtain the rest of the hydrodynamical variables for the $(\mathrm{r}, \mathrm{u})$ pairs previously found.

The LHS of equation (40) has to satisfy simultaneously the physical constraint $R \geq 1$ with $R$ a monotonically increasing function of $y$, and the algebra imposed by the RHS; however, this not possible for all $r>r_{\mathrm{sc}}$ when $V_{\mathrm{e}} \geq V_{\mathrm{e} \text {,pour }}$, and thus, the stationary solution in the outer zone does not exist when the last inequality is satisfied.

\section{THE GENERAL SOLUTION FOR THE FREE WIND ON EXTENDED HALOES}

Now, we proceed to present the model for an external superwind under the influence of a massive external halo. When external haloes are included, the equation of conservation of energy outside of the galaxy characteristic radius, equation (9), transforms into

$\frac{1}{r^{2}} \frac{\mathrm{d}\left[\rho u r^{2}\left(\frac{1}{2} u^{2}+(\eta+1) \frac{P}{\rho}\right)\right]}{\mathrm{d} r}=-\rho u\left(\nabla \phi+\nabla \phi_{\mathrm{h}}\right)$.

Above, the hydrodynamical variables are represented by their usual symbols, $\eta$ is the polytropic index and $-\nabla \phi=$ $-G M_{\mathrm{DM}} / r^{2}$, where $M_{\mathrm{DM}}$ is the total dynamical mass within $r_{\mathrm{sc}}$. Similarly, $-\nabla \phi_{\mathrm{h}}=-G M_{\mathrm{h}}(r) / r^{2}$, where $M_{\mathrm{h}}(r)$ is the cumulative dynamical mass (i.e. DM+BM) of the external halo, which has a total mass $M_{\mathrm{H}}$. We will allow the profile of the external halo to be defined either as a continuation of the internal profile or as a centrally truncated profile with different characteristics.

When $\nabla \phi=0$ our equations are analogous to the equations of Wang (1995). However, he considered only powerlaw and logarithmic gravitational potential finding a solution in terms of the Mach number $M$ as in the CC85 model. Here we consider general halo profiles (i.e. general gravitational potentials) and solve the equations in terms of the velocity giving explicit thresholds for the open-box enrichment regime (which Wang identified as a "galactic fountain" regime) and the obtention of accelerating superwind solutions. 
The integration of equation (44) yields a Bernoulli-like equation

$\frac{1}{2} u^{2}+(\eta+1) \frac{P}{\rho}=\frac{1}{2} V_{\mathrm{g}}^{2}-\tau \phi-\phi_{\mathrm{h}}$,

where $\phi=-G M_{\mathrm{DM}} / r, \phi_{\mathrm{h}}(r)$ is the gravitational potential at $r>r_{\mathrm{sc}}$ associated to the non-truncated version of the external halo, $\tau=1-M_{\mathrm{h}}\left(r_{\mathrm{sc}}\right) / M_{\mathrm{DM}}$ accounts for truncation effects, and $V_{\mathrm{g}}$ is the asymptotic terminal speed, which is given this time by

$\frac{1}{2} V_{\mathrm{g}}^{2}=\frac{1}{2} V_{\infty}^{2}-\frac{\tau G M_{\mathrm{DM}}}{r_{\mathrm{sc}}}+\phi_{\mathrm{h}}\left(r_{\mathrm{sc}}\right)-\frac{\left(1+\frac{1}{A}\right)}{(5-2 \alpha)} \frac{G M_{\mathrm{DM}}}{r_{\mathrm{sc}}}$.

Note that $\tau=0$ implies an uninterrupted, continuous gravitational potential. Similarly, $\tau<0$ implies a centrally truncated external halo with a mass larger than $M_{\mathrm{DM}}$, and $0<\tau<1$ implies the opposite. When $\tau=1$ there is no external halo, and thus $\phi_{\mathrm{h}}$ is identically zero.

We will work again in terms of dimensionless variables. For the present case they are:

$R=\frac{r}{r_{\mathrm{sc}}}, U=\frac{u^{2}}{V_{\mathrm{g}}^{2}}$, and $\Phi=-\tau \frac{V_{\mathrm{eg}}}{R}+\Phi_{\mathrm{h}}(R) ;$

where $V_{\text {eg }}$ is given by

$V_{\mathrm{eg}}=\frac{v_{\mathrm{e}}^{2}}{V_{\mathrm{g}}^{2}}=\frac{2 G M_{\mathrm{DM}}}{r_{s c} V_{\mathrm{g}}^{2}}$,

and $\Phi_{\mathrm{h}}(R)$ is $\phi_{\mathrm{h}}(r)$ written in terms of $R$ and normalized to $V_{\mathrm{g}}^{2} / 2$. The conservation laws can now be reduced to the same governing differential equation than in Section 3.2, see equation (32).

Thus, within the theoretical framework developed previously, it is very easy to prove that the supersonic free superwind solution is given by

$R=D U^{-1 / 4}\left[1-U+\tau \frac{V_{\mathrm{eg}}}{R}-\Phi_{\mathrm{h}}(R)\right]^{-\eta / 2}$,

with

$D=\left(\frac{1}{2 \eta+1}\right)^{1 / 4}\left\{\frac{2 \eta\left[1+\tau V_{\mathrm{eg}}-\Phi_{\mathrm{h}}(1)\right]}{2 \eta+1}\right\}^{\eta / 2}$.

Again, as previously, we will give preference to the parametric version of the solution:

$x\left[x-\tau V_{\mathrm{eg}}+R \Phi_{\mathrm{h}}(R)\right]^{\frac{2 \eta-3}{4}}=D_{0}(y+1)^{-\frac{1}{2 \eta+1}}(2 \eta-y)^{-\frac{2 \eta}{2 \eta+1}}$,

where $y$ is a parameter that varies between 0 and $2 \eta$ and

$x=R+\tau V_{\mathrm{eg}}-R \Phi_{\mathrm{h}}(R)$,

$y=\left[\frac{(2 \eta+1) U}{1+\tau \frac{V_{\mathrm{eg}}}{R}-\Phi_{\mathrm{h}}(R)}-1\right]$,

and

$D_{0}=\left[1+\tau V_{\mathrm{eg}}-\Phi_{\mathrm{h}}(1)\right](2 \eta)^{\frac{2 \eta}{2 \eta+1}}$.
To obtain the hydrodynamical profiles, one just needs to follow the algorithm presented at the end of Section 3.3. An advantage of the parametric solution is that it allows to work with just functions of $R$ in the first two critical steps, related to equations (51) and (52). On the other hand, equation (49) involves both $R$ and $U$. For $\eta=3 / 2$ (equivalent to the case $\gamma=5 / 3$ ) there is no need for a numerical root finder in the first step of our algorithm. In the second step however, its use will be most likely unavoidable, as the particular form of the assumed gravitational potential (i.e. of the external halo profile) is involved.

In Section 5.1, we will give the limit above which the stationary solution is disrupted in the external zone $\left(r>r_{\mathrm{sc}}\right)$ and the necessary condition for an accelerating stationary superwind solution for the case of extended haloes. In order to do this, we will specify first the normalized potential $\Phi_{\mathrm{h}}$ in the next section.

\section{THE EXTENDED HALO PROFILES}

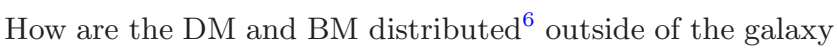
characteristic radius? Since we have permitted centrally truncated profiles for the external halo, theoretically, we can choose practically any of the usually assumed distributions; e.g. a NFW profile, Navarro, Frenk \& White (1997); a generalized NFW profile, Moore et al. (1999); a self-similar profile, Yoshikawa \& Suto (1999); an isothermal profile, and so on. Given that the most commonly used profiles depend on at least two parameters, and given also the additional freedom introduced by our truncated halo scheme; there is a vast number of profiles and parameters that can give reasonable agreement with observational studies and with the predictions of cosmological simulations.

We will try to rely on physical insight for selecting the external halo profile that we will use in our model. Recent cosmological simulations carried out by Abadi et al. (2010) predict that dark matter haloes always contract as a result of galaxy formation. They also found that the contraction effect is substantially less pronounced than predicted by the adiabatic contraction model (Blumenthal et al. 1986). On similar grounds, according to the high-resolution N-body cosmological simulations of $\Lambda \mathrm{CDM}$ haloes carried out by Navarro et al. (2010), the departures from similarity in the velocity dispersion and density profiles correlate in such a way, that a power law for the spherically averaged pseudophase-space density is preserved, $\rho / \sigma^{3} \propto r^{-1.875}$. They remarked that the index of the previous power law is identical to that of a Bertschinger's similarity solution for self-similar infall onto a point mass (in an Einstein-de Sitter Universe). They conclude that $\Lambda$ CDM haloes are not strictly universal, but that the departure from similarity previously mentioned may be a fundamental structural property.

Bearing in mind the results described above, we conclude that the cases $\tau<0$ and $0<\tau<1$ correspond to mathematically induced constraints that make continuous the potential at $r=r_{\mathrm{sc}}$ for arbitrarily-chosen external-halo profiles [see equation (45)]. This in turn might correspond

6 we will assume that they together can be specified by a single distribution profile $\rho_{\text {ext }}$. 
to an external haloe contracted (or expanded) just at the central object edge. The case $\tau=1$ corresponds to the case with no external halo. The case $\tau=0$ is of special interest, as it implies an unforced continuity of the gravitational potential. We will focus on this last case as it turns out that adequately chosen truncated Dehnen profiles satisfy naturally the latter condition.

For $r<r_{\mathrm{sc}}$, the cumulative dynamical mass corresponding to a truncated Dehnen profile ${ }^{7}$ is given by equation (13)

$M(r)=M_{\mathrm{DM}}(1+A)^{3-\alpha}\left(\frac{R}{R+A}\right)^{3-\alpha}$.

We will also assume a truncated Dehnen profile for the external halo, but we will demand a cumulative mass of the form:

$M_{\mathrm{h}}(r)=M_{\mathrm{DM}}\left(1+A_{1}\right)^{3-\alpha_{1}}\left(\frac{R}{R+A_{1}}\right)^{3-\alpha_{1}}$.

At $R=1$ we have that $M(1)=M_{\mathrm{h}}(1)$. Note that for this, we do not require $A_{1}=A$ nor $\alpha_{1}=\alpha$. The last property can be interpreted in terms of a contraction of an initial spatial configuration of DM and BM with concentration $A_{1}$ and steepness $\alpha_{1}$ which produced a new configuration with concentration $A$ and steepness $\alpha$ for $r<r_{\mathrm{sc}}$, or well, vice-versa, if other processes were involved (v.gr. angular momentum). On the other hand, a trivial but important relationship can be obtained from the condition $M(1)=M_{\mathrm{h}}(1)$ by separating the baryonic and dark matter components:

$r_{\mathrm{sc}}\left[M_{\mathrm{bar}}+M_{\mathrm{dark}}\right]=r_{\mathrm{sc}}\left[M_{\mathrm{bar}}+M_{\mathrm{dark}}\right]_{1}$.

This could be interpreted as an integral equivalent of the equation for adiabatic collapse derived by Blumenthal et al. (1986). Additionally, given that the radial velocity dispersion associated to the Dehnen profile goes as $\sigma \sim r^{\alpha / 2}$ when $r \rightarrow 0$, we are able to recover the index of the Bertschinger's power law near the centre of the galaxy when $\alpha=3 / 4$. However, Navarro et al. (2010) obtained the index from radial averaging, which implies that $\alpha$ can adopt values within a wider range.

Note that in turn, the previous configurations could be interpreted as the result of the contraction of an unperturbed configuration away from the galaxy. This is equivalent to saying that a galaxy formed from the perturbation of an initial state $\left(A_{0}, \alpha_{0}\right)$, and that after certain time, the perturbation bifurcated and produced two inner contracted states characterized by $(A, \alpha)$ and $\left(A_{1}, \alpha_{1}\right)$. The first state characterizes the inner regions of the galaxy, $r<r_{\mathrm{sc}}$. Then, the characteristic radius $r_{\mathrm{sc}}$ can be taken either as the radius of a galaxy nucleus or of a bulge. The second state characterizes the outer portions of the galaxy (e.g. a disc + DM). This is in agreement with the aforementioned cosmological simulations, and it implies that galaxies carved out gravitational potential holes when they formed and that they correspond to local depressions of an otherwise smoother gravitational potential.

Here, we are just interested in the superwind solution,

7 See also equation (3) in Dehnen (1993). so, in order to keep things simple, we will just consider the states $(A, \alpha)$ and $\left(A_{1}, \alpha_{1}\right)$, i.e. we will ignore the depression of the reference gravitational potential $\left(A_{0}, \alpha_{0}\right)$. The price that we will pay for this, as well as for the joint distribution of the baryonic and DM components assumed in our scheme, is that instead of (almost) 'perfectly' flat rotation curves up to 15 times the optical radius (Persic et al. 1996, Salucci \& Persic 1997), the rotation curves will show some downwards skewness at large radii. They are however very well above the curves corresponding to keplerian rotation of baryonic mass. Evenmore, the behaviour of the associated rotation curves away from $r_{\mathrm{sc}}$ is consistent with that of the universal rotation curves derived by Salucci et al. (2007) for spiral galaxies. Anyway, for our purposes, the behaviour at large radii is not that important, as the thermalization driven superwind solution is valid only close to the galaxy ${ }^{8}$ (see e.g. Strickland $\&$ Heckman 2009). So, we will proceed to give the expression corresponding to the external gravitational potential.

By taking the limit $R \rightarrow \infty$ in equation (55), one finds that the total dynamical mass is given by $M_{\mathrm{t}}=$ $M_{\mathrm{DM}}\left(1+A_{1}\right)^{3-\alpha_{1}}$. The expression of the associated gravitational potential for $0 \leq \alpha \leq 1$ is then similar to that given by equation (2) in Dehnen (1993):

$\Phi_{\mathrm{h}}(R)=-\frac{V_{\mathrm{eg}}\left(1+A_{1}\right)^{3-\alpha_{1}}}{\left(2-\alpha_{1}\right) A_{1}}\left[1-\left(\frac{R}{R+A_{1}}\right)^{2-\alpha_{1}}\right]$.

With this, we can establish new approximated thresholds for the open-box enrichment scenario and for accelerating superwind solutions.

\subsection{Thresholds for open-box enrichment and accelerating superwind solutions}

From the energy conservation law, it follows that when the effect of the external halo is considered, the asymptotic terminal speed is given by

$V_{\mathrm{g}}=\left[1-\left(\frac{1+\frac{1}{A}}{5-2 \alpha}\right) V_{\mathrm{e}}+\frac{V_{\mathrm{g}}^{2}}{V_{\infty}^{2}} \Phi_{\mathrm{h}}(1)\right]^{1 / 2} V_{\infty}$

The flow enters into non-stationary regimes (inpouring or outpouring) when

$\left(\frac{1+\frac{1}{A}}{5-2 \alpha}\right) V_{\mathrm{e}}-\frac{V_{\mathrm{g}}^{2}}{V_{\infty}^{2}} \Phi_{\mathrm{h}}(1) \geq 1$.

When the above inequality holds, the galaxy can eventually enter into an open-box enrichment scenario. Otherwise, we will have fully stationary solutions, unless radiative cooling or self-gravitation inhibit the stationary solution.

Fully stationary superwinds have accelerating velocity profiles when

$-(2 \eta+1) \frac{V_{\mathrm{g}}^{2}}{V_{\infty}^{2}} \Phi_{\mathrm{h}}(1)+2 \eta\left(\frac{1+\frac{1}{A}}{5-2 \alpha}\right) V_{\mathrm{e}} \leq 2 \eta$,

otherwise, they have decelerating velocity profiles. When the

8 This implies that the effect of the 'real' $\Phi_{\mathrm{h}}$ can be emulated there by giving adequate values to $A_{1}$ and $\alpha_{1}$. 
equality holds in the above relation, we have an almost constant external velocity profile with characteristic velocity

$$
V_{\mathrm{g}}=(2 \eta)^{-1 / 2}\left[-2 \phi_{\mathrm{h}}\left(r_{\mathrm{sc}}\right)\right]^{1 / 2}=
$$

$\eta^{-1 / 2} v_{\operatorname{rot}}\left\{\frac{\left(1+A_{1}\right)^{3-\alpha_{1}}}{\left(2-\alpha_{1}\right) A_{1}}\left[1-\left(\frac{1}{1+A_{1}}\right)^{2-\alpha_{1}}\right]\right\}^{1 / 2}$,

where $v_{\text {rot }}$ is the rotation speed at $r_{\mathrm{sc}}$.

\section{THE HYDRODYNAMICAL PROFILES}

\subsection{Superwinds on compact haloes}

We will discuss the effect of concentrated dynamical mass distributions with analogue mass and energy injections on the hydrodynamics using the reference models presented in Table 1, which condenses several important cases.

Models 1 and $\mathbf{2}$ correspond to synthetic SCUBA sources studied by Silich et al. (2010) using their numerical eulerian code, which incorporates both the gravitational field and radiative cooling. They assumed a uniform distribution of the protogalaxy parameters. They also considered a continuous star formation scenario and that mass loading was proportional to 0.5 times the SFR. According to the Strickland \& Heckman (2009) definition of mass loading, one would require a coefficient $\beta \sim 5.77$ in order to obtain the value of $V_{\infty}$ used by Silich et al. (2010). Model 3 is identical to Model 1, but this time, we have assumed a plateau-like distribution $(\alpha=0)$ with concentration parameter $A=0.546$. The corresponding velocity profiles are shown in Fig. 7. There, the profiles for the external zone were obtained analytically. Because of the effect of the gravitational field, all models have asymptotic terminal speeds significantly less than $V_{\infty}=1144.6 \mathrm{~km} \mathrm{~s}^{-1}$. For models 1 and 2, we reproduce the numerical results obtained by the previous authors. Note that as predicted in Appendix B, radiative losses are negligible. In addition, as predicted by equation (21), Model 1 has a bounded accelerating profile and Model 2 a bounded decelerating one. Silich et al. (2010) reported a terminal speed of $\sim 740 \mathrm{~km} \mathrm{~s}^{-1}$ for Model 1 (the value of $u$ at $r \approx 10 \mathrm{kpc}$ ). Using equation (20), we find that actually, $V_{\mathrm{g}} \approx 697 \mathrm{~km} \mathrm{~s}^{-1}$. Similarly, $V_{\mathrm{g}} \approx 246 \mathrm{~km} \mathrm{~s}^{-1}$ for Model 2 and $V_{\mathrm{g}} \approx 479 \mathrm{~km} \mathrm{~s}^{-1}$ for Model 3. This last model reflects the impact of the distribution assumed for the protogalaxy parameters. We obtain an almost constant velocity profile when the dynamical mass and the energy and mass injections are more concentrated towards the object centre, as indicated in Table 1.

Model 4 is based on parameters fitted to the nucleus of the dwarf elliptical galaxy FCC 303 by Hilker et al. (2007, see also Turner et al. 2012). In the optical, this object is among the brightest in the central region of the Fornax Cluster and has a central surface brightness profile similar to those of ultra-compact dwarf galaxies (UCDs). Hilker et al. (2007) used high resolution spectroscopy and surface brightness modeling techniques to derive a dynamical mass, mass-to-light ratio, cut-off radius and half-light radius that are consistent with virial estimators. They concluded that the mass-to-light ratio of FCC 303 is entirely compatible

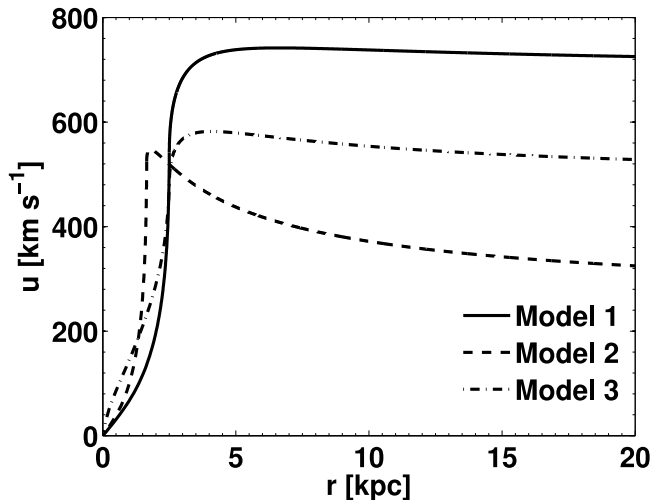

Figure 7. The velocity profile for the models representing SCUBA sources. Here, the profiles for $r>r_{\mathrm{sc}}$ were obtained analytically.

with a pure stellar population, i.e. no dark matter is required in order to explain it. Model 5 tries to emulate the most extreme values of the dynamical mass, radius and SFR presented by Peeples et al. (2008) for a sample 43 isolated galaxies with morphologies similar to $\mathrm{dE}$ and $\mathrm{dSph}$, lowmasses $\left(M_{\mathrm{DM}} \sim 10^{7.4}-10^{10} \mathrm{M}_{\odot}\right)$ and high-oxygen content $(8.95<12+\log (O / H)<9.3)$. The correspondence between our adopted values and those in their sample is not one-toone though, since we have adopted a rather small radius for the most massive galaxy. This was necessary because of the analogue injection of mass and energy in our hydrodynamical model. Finally, Model 6 presents the case of an artificial massive BCD-like galaxy.

For all these models, we have assumed a generic terminal speed of $V_{\infty}=2500 \mathrm{~km} \mathrm{~s}^{-1}$ with $\beta=1$ and $\epsilon=1$. This value fairly agrees with the superwind recipe given by Strickland \& Heckman (2009) for models that depart from a fixed velocity; however, here we are considering a different hydrodynamical setting than in their work. So, afterwards, we adjusted the values of $\beta$ and $\epsilon$ to obtain effective terminal speeds around $V_{\infty} \sim 1000 \mathrm{~km} \mathrm{~s}^{-1}$, in order to get asymptotic terminal speeds that are consistent with the observed typical values (of hundreds of kilometers). This was done with the aim of studying if typical outflows can be produced by objects with characteristics similar to those present in models $4-6$, at a time at which they could have been experiencing moderate starburst activity for their type.

In Fig. 8, we present the velocity, temperature and density profiles corresponding to models 4,5 and 6 . For model 4 , which has a moderate concentration parameter, the external profiles are very similar to the ones predicted by the CC85 model, since they are barely affected by the gravitational field produced by the relatively low mass of the galaxy, in spite of the assumed high mass-loading. As a consequence, the superwind reaches an asymptotic terminal speed similar to the effective, $V_{\mathrm{g}} \sim V_{\infty}$. This is consistent with the view that outflows can more easily remove material from the least-massive galaxies. However, we found that the central density is larger than the one predicted by the CC85 model by a factor of $\sim 2$. This implies that the assumed concentration of the starburst will produce a brighter diffuse emission from the hot gas that remains inside the galaxy. The internal temperature profile is also somewhat steeper than that predicted by the CC85 model. 

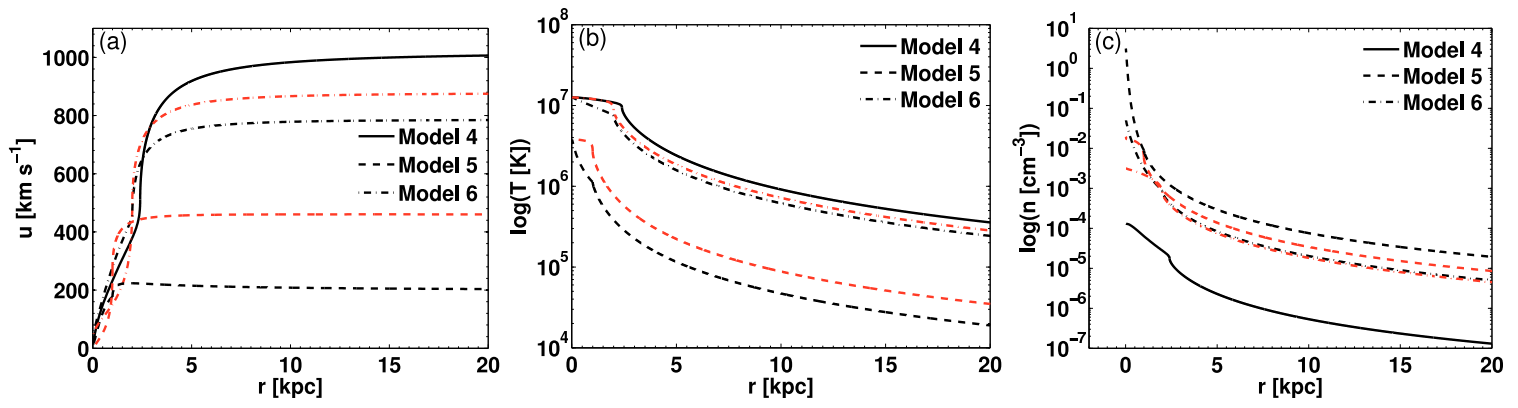

Figure 8. The velocity, temperature and number density profiles for an object with mass, radius and concentration similar to those of FCC330 (model 4), an isolated dE or dSph galaxy with low-mass and high concentration parameter (model 5), and an artificial high-mass BCD (model 6). The red lines trace the expected profiles for models 5 and 6 if a uniform distribution is assumed.

Model 5 is the one that deviates the most from the predictions that can be extracted from a uniform distribution of the relevant parameters. The asymptotic terminal speed is now $\sim 0.35 V_{\infty}$ instead of being almost the same. Consequently, the temperature of the associated bubble (Weaver et al. 1977) would be reduced by more than $85 \%$ and its growing would be compromised. The characteristics of the superwind emission are also drastically changed. The central temperature is identical to the one derived from a uniform distribution, but at intermediate and large radii it is inferior by a factor of $\sim 2$. Most remarkably, the central density is higher by more than two orders of magnitude and the density profile is very steep. This will translate into much brighter but compact cores. At large $r$ the density differs from the predictions of the uniform distribution by a factor $\sim 2$. Model 5 is at the skirts of the limit for inpouring or outpouring, and very close to becoming radiatively unstable. A slightly higher concentration will certainly make it enter into the open-box or even the closed-box enrichment scenario. On the other hand, a larger SFR would make it enter in a catastrophic cooling regime.

In Model 6, the velocity profile is flattened by the effect of the large total dynamical mass and its steeper distribution. The resulting reduction of the asymptotic terminal velocity with respect to the effective terminal speed, will in turn reduce the post-shock temperature expected from the standard bubble model by $\sim 40 \%$. The external temperature and density profiles and the internal temperature profile somewhat differ from the ones that would result if one assumes a uniform distribution; nevertheless, drastic differences exist in the internal velocity and density profiles. The densities within the innermost regions of the galaxy are more than one order of magnitude larger than the values predicted by the uniform distribution. This is produced by a combination of compactness and a steeper dynamical mass profile. This will translate into an increase of the expected diffuse Xray emission (or in other bands provided that the luminosity in band b could be expressed as $\left.L_{\mathrm{b}} \propto \int \rho^{2} \Lambda_{\mathrm{b}} \mathrm{d} V\right)$ of the central regions by 1 to 2 orders of magnitude. This can render the luminosity of the free superwind at large $r$ dimmer in comparison and also harder to detect for a given detection threshold. The picture obtained from this synthetic model seems to be consistent with observations of BCDs.

Model 7 corresponds to one of the sets of parameters found by Strickland \& Heckman (2009) for M 82 through an extensive observational and theoretical study. As in their work, model 7 assumes a CC85 model for the hydrodynamics. Model 8 also corresponds to M 82, but it assumes a steeper distribution $(\alpha=1 / 2)$ and a concentration parameter that was derived from a smaller radius also used by Strickland \& Heckman (2009) to model this starburst galaxy. As in model 4, given the low mass of the galaxy, we do not obtain significant differences between these two models with respect to the CC85 solution, although a steeper distribution can compromise the solution stability.

\subsection{Superwinds on extended haloes}

In our model, the dynamical mass $\left(M_{\mathrm{DM}}\right)$ contained within a bulge or galaxy nucleus experiencing an starburst episode is related to the concentration parameter and steepness of the external halo and to the total dark matter and baryonic mass $M_{\mathrm{t}}$ :

$M_{\mathrm{DM}}=\frac{M_{\mathrm{t}}}{\left(1+A_{1}\right)^{3-\alpha_{1}}}$.

The dynamical mass contained in the external halo $(r>$ $\left.r_{\mathrm{sc}}\right)$ is

$M_{\mathrm{H}}=M_{\mathrm{t}}\left[1-\frac{1}{\left(1+A_{1}\right)^{3-\alpha_{1}}}\right]$.

Similarly, the dynamical mass contained up to an external characteristic radius (normalized to $r_{\mathrm{sc}}$ ), $R_{\mathrm{D}}$, is

$M_{\mathrm{D}}=M_{\mathrm{t}}\left(\frac{R_{\mathrm{D}}}{R_{\mathrm{D}}+A_{1}}\right)^{3-\alpha_{1}}$.

The radius $R_{\mathrm{D}}$ can be associated to a BM 'disc' radius or well to the $\mathrm{BM}+\mathrm{DM}$ virial radius. So, all the relevant galaxy parameters are correlated, in a similar fashion as in the work of Salucci et al. (2007). Nevertheless, we emphasize that the relationship between the parameters is alike but of course not the same, since here we constructed our theoretical model only following the results of the simulations of Abadi et al. (2010) and Navarro et al. (2010).

We will proceed to discuss the effect of the extended haloes on the hydrodynamics. In order to do this, we consider the hydrodynamical models presented in Table 2 and Table 3. The first table gives the inner parameters for three galaxies with different characteristics. The second table gives the properties of their external haloes. The groundwork for 
Table 1. Reference hydrodynamical models

\begin{tabular}{|c|c|c|c|c|c|c|c|c|c|c|c|c|}
\hline Model & Type & $\begin{array}{l}\alpha \\
\text { (a) }\end{array}$ & $\begin{array}{l}A \\
\text { (b) }\end{array}$ & $\begin{array}{l}r_{\mathrm{sc}} \\
(\mathrm{kpc}) \\
(\mathrm{c})\end{array}$ & $\begin{array}{l}M_{\mathrm{DM}} \\
\left(\times 10^{8} \mathrm{M}_{\odot}\right) \\
(\mathrm{d})\end{array}$ & $\begin{array}{l}\mathrm{SFR} \\
\mathrm{M}_{\odot} \mathrm{yr}^{-1} \\
\text { (e) }\end{array}$ & $\begin{array}{l}\beta \\
\text { (f) }\end{array}$ & $\begin{array}{l}\epsilon \\
(\mathrm{g})\end{array}$ & $\begin{array}{l}\zeta \\
(\mathrm{h})\end{array}$ & $\begin{array}{l}V_{\infty} \\
\mathrm{km} \mathrm{s}^{-1} \\
(\mathrm{i})\end{array}$ & $\begin{array}{l}V_{\mathrm{e}} \\
(\mathrm{j})\end{array}$ & $\begin{array}{l}\text { Regime } \\
(\mathrm{k})\end{array}$ \\
\hline 1 & SCUBA & 0 & $\infty$ & 2.5 & 2000 & 2 & $\approx 5.77$ & 1 & 1 & 1144.6 & 0.5246 & accelerating \\
\hline 2 & SCUBA & 0 & $\infty$ & 1.65 & 2000 & 2 & $\approx 5.77$ & 1 & 1 & 1144.6 & 0.7949 & decelerating \\
\hline 3 & SCUBA & 0 & 0.5364 & 2.5 & 2000 & 2 & $\approx 5.77$ & 1 & 1 & 1144.6 & 0.5246 & $\sim$ constant, $V_{\mathrm{g}}=u_{\mathrm{sc}}$ \\
\hline 4 & FCC 303 & 0 & 0.6 & 2.4 & 3.3 & 3 & 3 & 0.5 & 1 & 1021 & 0.0011 & accelerating \\
\hline 5 & $\mathrm{dE} / \mathrm{dSph}$ & 0 & 0.1 & 1 & 100 & 0.1 & 4 & 0.2 & 1 & 560 & 0.2740 & borderline \\
\hline 6 & $\mathrm{BCD}$ & 0.75 & 0.4 & 2 & 500 & 3 & 3 & 0.5 & 1 & 1021 & 0.2062 & accelerating \\
\hline 7 & M 82 & 0 & $\infty$ & 0.3 & 7 & 4.5 & 1.7 & 0.55 & 1 & 1426 & 0.0099 & accelerating \\
\hline 8 & M 82 & 0.5 & 0.42 & 0.3 & 7 & 4.5 & 1.7 & 0.55 & 1 & 1426 & 0.0099 & accelerating \\
\hline
\end{tabular}

Superwind hydrodynamical models for different kinds of galaxies. Table headers: (a) steepness parameter, (b) concentration parameter, (c) radius, (d) dynamical mass, (e) star formation rate, (f) mass loading factor, (g) thermalization efficiency, (h) participation factor (i) effective terminal speed, (j) squared ratio of the escape velocity to the effective terminal speed, (k) flow regime.

the discussion will be the premise that the spherical symmetric superwind solution is a zeroth-order approximation to the aspherical case. We will consider again a reference effective terminal speed of $2500 \mathrm{~km} \mathrm{~s}^{-1}$ for the case of null mass-loading, fully efficient thermalization, and total participation within the starburst volume, i.e. for $\epsilon=\beta=\zeta=1$. For models 2 and 3 , the SFRs were obtained from formula (1) in Rupke, Veilleux \& Sanders (2005a) and formula (B5), i.e. we considered SFRs that are consistent with the typical observed luminosities for the object types, and that in parameter space, place the objects below the threshold for catastrophic cooling. We find that the predicted temperature profile is barely modified by the presence of the extended haloes. However, drastic changes are produced in the velocity profile.

Model 1 is an extended version of model 5 in Table 1 , and corresponds to a synthetic isolated dwarf elliptical galaxy that tries to emulate the characteristics of the most massive outlier of the mass-metallicity relationship detected by Peeples, Pogge \& Stanek (2008). We assumed that the galaxy formed by a contraction of $\sim 40 \%$ of an initially unperturbed subhalo of DM and BM which had $\sim 70 \%$ of its total mass located within $r \sim 3 r_{\mathrm{sc}}$, so we used $A_{1}=0.5$, $\alpha_{1}=3 / 4$ and $R_{\mathrm{D}}=1$. The latter is equivalent to saying that in this case there is no disc, i.e. we only have a galaxy nucleus. The internal dynamical mass distribution follows a plateau-like profile, which implies that some mechanism - perhaps internal dynamical processes with the action of early powerful superwinds associated to a more extended and powerful starburst episode (see Governato et al. 2010) - has also transformed the initial mass configuration. In this model, starburst activity still persists near the galaxy centre, but with a high concentration. We assumed a low thermalization efficiency, which implies a small number of massive stars and SNe within the characteristic concentration radius, $A=0.1$. The justification for this is that the SFR is low, and that although small, the concentration radius is still much larger than the typical radius of a massive star, i.e. the filling factor is low. Similarly, because of the small number of massive stars, just a small incorporation of mass is necessary to produce a heavily mass-loaded superwind. In this model, the presence of the extended halo suppresses the free superwind solution and the galaxy experiences an open-box enrichment [see equation (59)] by keeping the metals processed by the few massive stars still present near the galaxy centre. This will require however an already gas-poor galaxy at the moment at which the pollution occurred (Peeples, Pogge \& Stanek, 2008). As suggested above, the required low mass fraction could have been produced by the action of early superwinds associated to previous and more powerful starburst activity. This is consistent with the views of Peeples et al. (2008), which regarded their sample of outliers as transitional galaxies in their way to becoming typically isolated $\mathrm{dE}$ and $\mathrm{dSph}$ galaxies, but with a high metallicity. The suppression of the free superwind solution is practically insensitive to the value of $0 \leq \alpha_{1} \leq 1$, which indicates that the enrichment is produced by the physical conditions within $A$ and the initial concentration of the unperturbed subhalo from which the galaxy formed.

Model 2 considers the synthetic and very massive blue compact dwarf galaxy modeled previously (see Table 1, Model 6). However, here we add an extended 'disc' to the model in order to 'transform' the galaxy into a luminous infrared one ${ }^{9}$ (LIRG, $\left.L_{I R} \sim 10^{11} \mathrm{~L}_{\odot}\right)$. LIRGs and ULIRGs may be the end result of the merging of two moderate-size spiral galaxies and display traces of convergence to an elliptical morphology (Sanders \& Mirabel 1996; Rupke et al. 2005a). We will model a LIRG assuming that it displays a morphology similar to that of the central component of Arp 299 (Sargent \& Scoville 1991; Heckman et al. 1999; Hibbard \& Yun, 1999; Hu et al. 2004), but with just one nucleus. We assume that the disc extends to up to 5 times the radius of the merger nucleus; thus, $R_{\mathrm{D}}=5$. The assumed mass and extension are consistent with $\mathrm{CO}$ emission observations of (U)LIRGs (Lonsdale, Farrah \& Smith 2006 and references therein). We further assume that the merging process has similarly transformed the steepnesses of the internal and external mass profiles of the interacting galaxies unperturbed haloes, such that $\alpha=\alpha_{1}=3 / 4$. We adopt the

9 N.B. As LIRGs and ULIRGs, BCDs may be the result of mergers, although generally they have lower masses, given that they mostly form from the merging of dwarf galaxies. Nevertheless, on a higher end, luminous blue compact galaxies can have dynamical masses of up to $\sim 10^{12} \mathrm{M}_{\odot}$ (Garland et al. 2004, Pisano et al. 2010). 


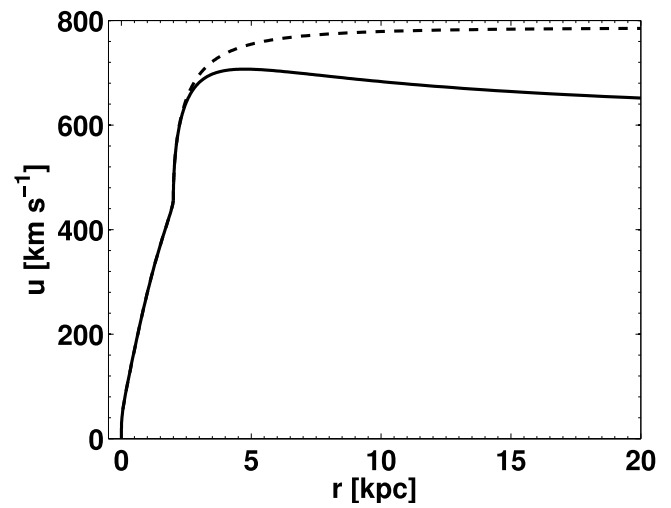

Figure 9. Superwind velocity profile for model 2 (solid line). The dashed-line represents the profile that would result if the external halo were neglected.

value $A_{1}=1$ since it produces interesting proportions. In such a case, $\sim 66 \%$ of the BM and DM of both galaxies is contained within the warped discs characteristic radius and about $\sim 30 \%$ of this fraction resides within the merger nucleus (that is $\sim 20 \%$ of the total mass). As in the original model, starburst activity is present in the nucleus with a somewhat high concentration $(A=0.4)$, the thermalization efficiency is 0.5 and mass loading is important, $\beta=3$ (see Heckman et al. 1999). In this model, the gravitational field of the external halo transforms the accelerating superwind solution associated to the original model into a bounded decelerating one (Fig. 9). This effect occurs because now we have a more massive galaxy. The produced deceleration will enhance the observable properties of the superwind because of a proportional density increment $\left(\rho \propto u^{-1}\right)$. However, an even larger total mass could result in the inhibition of the superwind solution. This is consistent with the superwind scaling properties found by Rupke, Veilleux \& Sanders (2005b), whom reported and initial increment of the superwind observable properties with galaxy mass and a posterior flattening with the same.

Rupke et al. (2005b) also reported a flattening of the superwind observable properties at high SFR. In principle, the normalized free superwind solution is insensitive to the SFR (provided that it could be considered constant during a relatively large time interval), as it just depends on the effective and asymptotic terminal speeds. However, high SFRs will intensify the effect of radiative cooling, as more mass will be injected per unit time and volume, and thus, the stationary solution could also be radiatively inhibited.

Model 3 gives an extreme example of the effect of the nominal value of the galaxy mass: we model a massive and 'rare' radio galaxy with a very extended halo (see e.g. Genzel et al. 2003). We consider a galaxy with a dynamical mass of $1 \times 10^{11} \mathrm{M}_{\odot}$ within its nucleus of $r_{\mathrm{sc}} \sim 2 \mathrm{kpc}$. A mildly concentrated starburst $(A=0.5)$ is present in the nucleus, which has a cuspy dynamical mass distribution $(\alpha=1)$. We consider that the steepnesses of the inner region and the halo are the same and that the total mass of the galaxy is $M_{\mathrm{t}}=4 \times 10^{11} \mathrm{M}_{\odot}$. This requires that $A_{1}=1$. This implies that the half-mass radius is $r \sim 2.5 r_{\mathrm{sc}}$ and that $\sim 80 \%$ of the total mass is contained within $r \sim 8 r_{\mathrm{sc}}$. In this model a high deceleration of the superwind is produced, and the flow is unstable to small variations of the effective terminal

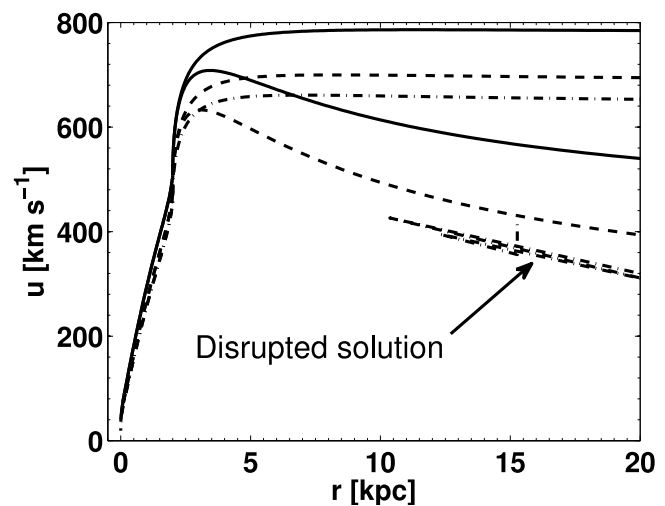

Figure 10. Velocity profiles for model 3. The solid lines corresponds to the parameters showed in Table 2. For this parameters, $V_{\infty} \approx 1208 \mathrm{~km} \mathrm{~s}^{-1}$. The lower (upper) solid line (does not) consider(s) the presence of the external halo. Similarly, the dashed and dash-dotted lines correspond to $V_{\infty}=1150 \mathrm{~km} \mathrm{~s}^{-1}$ and $V_{\infty}=1125 \mathrm{~km} \mathrm{~s}^{-1}$, respectively. For the latter case, the stationary free superwind solution does not exist.

speed (thermalization efficiency), as shown in Fig 10. As a consequence, the flow could eventually enter into the outpouring or even the inpouring regime. On the other hand, if instead of a continuous steepness, we consider that the typical cuspy halo profile (with slope $\alpha=1$ ) resulted from the contraction of a smoother one, say with $\alpha_{1}=3 / 4$ and $A_{1}=1$, the free superwind solution would be inhibited and the galaxy could enrich itself with is produced metals in an open-box scenario. This would occur because in the second case, the total mass is slightly larger, $M_{\mathrm{t}}=4.75 \times 10^{11} \mathrm{M}_{\odot}$. The cumulative dynamical masses of the two assumed external profiles are very similar, their ratio varies from a value of 1 at $r_{\mathrm{sc}}$ (they are identical as they must), up to a value $\sim 0.86$ at $r=10 r_{\mathrm{sc}}$; nevertheless, such a small variation is enough to suppress the stationary superwind solution. This reflects the fact that at the limit of large galaxy masses, galaxies will retain most of their metals, as expected.

We next present a comparison with observational data and a discussion of the implications of these results for the mass-metallicity relationship.

\section{DISCUSSION AND COMPARISON WITH OBSERVATIONAL DATA}

In panel (a) of Fig. 11, we present the effective terminal speeds derived by Heckman et al. (2000) from X-rays and $\mathrm{Na} \mathrm{D}$ absorption-lines observations of superwinds in nearby galaxies. As a reference, these authors included the lines $v_{\mathrm{e}}=2 v_{\text {rot }}$ and $v_{\mathrm{e}}=3 v_{\text {rot }}$. Their data suggest that superwinds escape only from galaxies with small rotation speeds, which in turn indicates shallow gravitational potentials and low masses. In panel (b) of the same figure, we present the lines above which accelerating superwind solutions can be obtained for different concentration parameters and steepnesses. This lines come from equation ${ }^{10}(21)$, and we have assumed a plateau-like and a Herquist-like profile. The latter reproduce the behaviour of cuspy dark matter haloes.

${ }^{10} V_{\infty}^{2}=2 v_{\text {rot }}^{2} / V_{\mathrm{e}, \mathrm{cons}}$. 
Table 2. Reference hydrodynamical models. Galaxy parameters for $r<r_{\mathrm{sc}}$.

\begin{tabular}{|c|c|c|c|c|c|c|c|c|c|c|c|c|}
\hline Model & Type & $\begin{array}{l}\alpha \\
\text { (a) }\end{array}$ & $\begin{array}{l}A \\
\text { (b) }\end{array}$ & $\begin{array}{l}r_{\mathrm{sc}} \\
(\mathrm{kpc}) \\
(\mathrm{c})\end{array}$ & $\begin{array}{l}M_{\mathrm{DM}} \\
\left(\times 10^{8} \mathrm{M}_{\odot}\right) \\
(\mathrm{d})\end{array}$ & $\begin{array}{l}\mathrm{SFR} \\
\mathrm{M}_{\odot} \mathrm{yr}^{-1} \\
(\mathrm{e})\end{array}$ & $\begin{array}{l}\beta \\
\text { (f) }\end{array}$ & $\begin{array}{l}\epsilon \\
(\mathrm{g})\end{array}$ & $\zeta$ & $\begin{array}{l}V_{\infty} \\
\mathrm{km} \mathrm{s}^{-1} \\
\text { (i) }\end{array}$ & $\begin{array}{l}V_{\mathrm{e}} \\
(\mathrm{j})\end{array}$ & $\begin{array}{l}\text { Regime (No halo) } \\
(\mathrm{k})\end{array}$ \\
\hline 1 & $\mathrm{dE}$ & 0 & 0.1 & 1 & 100 & 0.1 & 4 & 0.2 & 1 & 560 & 0.2740 & borderline \\
\hline 2 & (L)BCD/LIRG & 0.75 & 0.4 & 2 & 500 & $\sim 40$ & 3 & 0.5 & 1 & 1021 & 0.2060 & accelerating \\
\hline 3 & Radio & 1 & 0.5 & 2 & 1000 & $\sim 200$ & 3 & 0.7 & 1 & 1208 & 0.2946 & accelerating \\
\hline
\end{tabular}

Superwind hydrodynamical models. Table headers: (a) steepness parameter, (b) concentration parameter, (c) radius, (d) dynamical mass, (e) star formation rate, (f) mass loading factor, (g) thermalization efficiency, (h) participation factor (i) effective terminal speed, (j) squared ratio of the escape velocity to the effective terminal speed, and (k) flow regime when the external halo is neglected.

Table 3. Reference hydrodynamical models. External halo parameters.

\begin{tabular}{|c|c|c|c|c|c|c|c|}
\hline Model & Type & $\begin{array}{l}\alpha_{1} \\
\text { (a) }\end{array}$ & $\begin{array}{l}A_{1} \\
\text { (b) }\end{array}$ & $\begin{array}{l}r_{\mathrm{D}} \\
(\mathrm{kpc}) \\
(\mathrm{c})\end{array}$ & $\begin{array}{l}M_{\mathrm{D}} \\
\left(\times 10^{8} \quad \mathrm{M}_{\odot}\right) \\
(\mathrm{d})\end{array}$ & $\begin{array}{l}M_{\mathrm{t}} \\
\left(\times 10^{8} \mathrm{M}_{\odot}\right) \\
(\mathrm{e})\end{array}$ & $\begin{array}{l}\text { Regime } \\
\text { (f) }\end{array}$ \\
\hline 2 & LIRG & 0.75 & 1 & 5 & $\sim 3.16 M_{\mathrm{DM}}$ & $\sim 4.76 M_{\mathrm{DM}}$ & decelerating \\
\hline 3 & Radio & $1(3 / 4)$ & 1 & $R_{\mathrm{hm}}=2.5$ & $2 M_{\mathrm{DM}}$ & $4 M_{\mathrm{MD}}$ & decelerating (open-box enrichment) \\
\hline
\end{tabular}

External halo parameters for the models presented in Table 1. Table headers: (a) steepness parameter, (b) concentration parameter, (c) 'disc' radius (d) 'disc' mass, (e) total mass, and (f) Regime. In model $3, R_{\mathrm{hm}}$ corresponds to the half-mass radius.

The former is more adequate to model the matter distribution of dwarf galaxies. The dashed and dotted lines represent thresholds for the effective terminal speed. Thermalization and mass-loading inside of a (proto-) galaxy have to occur in such a way that $V_{\infty}$ has to be larger than the respective threshold value if an accelerating superwind is to be produced.

As it is shown in Fig. 11, the higher the concentration (i.e. for smaller $A$ ) the more stringent are the requirements for producing an outflow, as lower mass injections, poor mass-loading and a higher and more efficient energy injection inside of galaxies would be necessary in order to reach the needed value of $V_{\infty}$. At first sight, the sample of Heckman et al. (2000) seems to correspond to galaxies with intermediate concentrations, given that the line $v_{\mathrm{e}}=2 v_{\text {rot }}$ in their figure is similar to the lines for $A=0.5$. However, some caution needs to be exerted. As Heckman et al. (2000) have indicated, for the X-rays data, they adopted a conservative approach in deriving the effective terminal speed by associating the observed X-ray temperature to the central temperature predicted by the CC85 model. In our model, the X-ray temperature is between the value derived by Heckman et al. (2000), equation (26), and a smaller value that depends on the intensity of the gravitational field and on the kinetic energy, equation (25). This effect can be relevant for massive galaxies, specially given that projection effects will in turn determine the observed value of the X-ray temperature (Añorve-Zeferino et al. 2009). However, when pertinent, the consideration of such effects would only displace the Xray data in Fig. 11 upwards. This will reflect the fact that deeper gravitational potentials also impose a more stringent condition over the required value of $V_{\infty}$ and will provide additional support for the Heckman et al. (2000) conclusion that superwinds can remove metals more easily from the least-massive galaxies.
On the other hand, the Na D data is more suitable for representing the asymptotic terminal speed. In Fig. 11b we also display the expected value of its threshold, $V_{\mathrm{g}, \text { th }}$, equation (22). One must remind that such threshold depends just on $v_{\text {rot }}$. Both the $\mathrm{Na} \mathrm{D}$ and the $\mathrm{X}$-rays data are completely above this limit. This is exactly what should be expected according to our model and the observed lack of correlation between the velocity dispersion in the absorbing material with the galaxies rotation speed (Heckman et al. 2000): if the effective terminal speed satisfies the requirements imposed by gravity and the concentration of the starburst for an accelerating solution $\left(V_{\mathrm{e}}<V_{\mathrm{e}, \mathrm{cons}}\right)$, the resulting superwind will reach an asymptotic terminal speed $V_{\mathrm{g}}>V_{\mathrm{g}, \mathrm{th}}$ and no correlation with the galaxy rotation speed will be observed. However, one could have expected that departures from spherical symmetry, local effects, the presence of a disc, and all the usual 'buts' would have produced at least some deviations. It seems withal that the threshold $V_{\mathrm{g}, \text { th }}$ is a reliable lower limit.

Then again, the difficulties imposed by highly concentrated starburst for outflows can be an argument for explaining the high metallicity measured by Peeples et al. (2008) for their sample of low-mass outliers of the M-Z relationship. The galaxies in their sample do not occupy unexpected positions in the color-magnitude diagram, have normal SFRs and are not unusually compact. They point out that the only remarkable morphological characteristic in their sample is the presence of bright and often very blue compact cores in ten of the galaxies. They also suggest that their sample represents transitional dwarf galaxies at the end of their star formation activity and at the edge of becoming typical isolated $\mathrm{dE}$ and $\mathrm{dSph}$ galaxies. However, their high metallicities do not correspond to the expectations for BCDs. Since the galaxies they selected are isolated and non-interacting, environmental effects can be discarded (Ellison et al. 2009). 

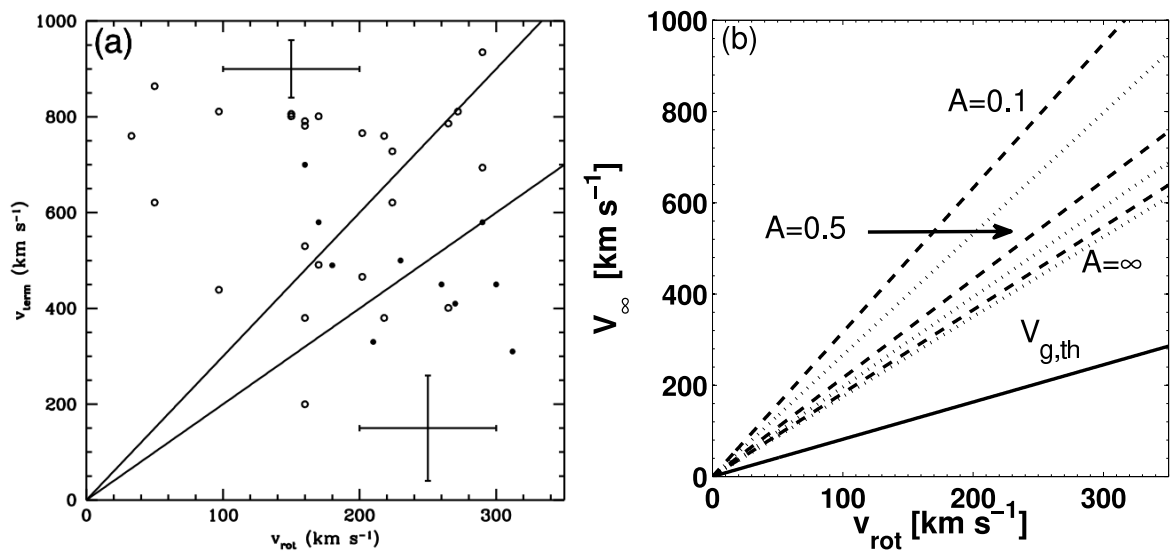

Figure 11. Panel (a): fig. 12 from Heckman et al. (2000), reproduced by permission of the AAS. The figure shows the galaxy rotation speed vs. the inferred terminal speeds of superwinds produced by nearby galaxies. The terminal speeds were derived by Heckman et al. (2000) from X-ray (hollow dots) and Na D (solid dots) observations. The two diagonal lines indicate the galaxy escape velocity under the assumption that $v_{\mathrm{e}}=2 v_{\mathrm{rot}}$ and $v_{\mathrm{e}}=3 v_{\text {rot }}$. The lower (upper) error bar indicates the typical uncertainties in the X-ray (Na D) estimations of $V_{\infty}$. Panel (b): threshold lines for the effective terminal speed needed to establish an accelerating superwind solution as a function of the galaxy rotation speed. The assumed concentration parameters are indicated in the figure. The dashed lines correspond to $\alpha=1$ and the dotted lines to $\alpha=0$. The solid line indicates the minimum asymptotic terminal speed that an accelerating outflow must reach for a given rotation speed.

In terms of a closed-box enrichment model, Peeples et al. (2008) explain that the only possibility is that these galaxies might have low gas fractions for their masses. In such a case, only a small pollution would be enough to enrich the gas (Dalcanton 2007). On similar grounds, Ellison et al. (2008) analyzed a large galaxy sample from SDSS and determined that at fixed mass, galaxies with smaller half-light radii tend to have higher abundances. They proposed that superwinds could be responsible of the selective loss of metals.

We also suggest that the above effects are or were produced by superwinds struggling against the sharp central gravitational potential of the concentrated starburst episodes, which might correspond to blue cores. The model here presented (see Model 1 in Section 6.2) can provide a basis to qualitatively explain these results, as well as to quantitatively evaluate if the superwind hypothesis can approximate the observed dispersion of the mass-metallicity relationship (Tremonti et al. 2004).

Finally, we remark that our model has the scaling properties reported by Rupke et al. (2005b). It has inherited the scaling with star formation rate and effective terminal speed of the CC85 model. On the other hand, its scaling properties with respect to galaxy mass and radius are different because of the incorporation of the gravitational field. For a fixed SFR, Rupke et al. (2005b) reported the enhancement of the superwind properties with increasing galaxy mass as an unexpected result. We have shown that at fixed SFR and starburst concentration, this can be explained in terms of the larger densities of the outflows associated to the most massive galaxies; so, the larger the galaxy mass the more intense the observable manifestations of the superwind. However, this cannot continue indefinitely, as equations (19) and (59) establish limits above which galactic superwinds eventually enter into the inpouring or outpouring regimes. In turn, this can explain the observed flattening of the observ- able superwind properties for large galaxy masses and SFRs also reported by Rupke et al. (2005b). As an alternative to explain the flattening, those authors proposed heuristically the existence of a terminal velocity for ULIRGs above which superwinds cannot be accelerated, and/or a reduction in thermalization efficiency at high SFR. According to our model, we find the opposite: there exist an asymptotic effective terminal velocity below which no accelerating superwind exists and such velocity depends on the galaxies rotation speed (or mass and radius), concentration of their starburst episode, and the steepness of the distribution of their dynamical mass, see equations (21) and (60).

\section{CONCLUSIONS}

In this theoretical work we presented an analytical model that permits to predict the impact of the the gravitational field on the free superwind stationary solution. Our general findings are:

(i) The existence or inhibition of the stationary superwind solution highly depends on the concentration and steepness of the dynamical mass and mass and energy injection rates. A superwind can be more easily inhibited when the steepness and concentration are high.

(ii) We found that the gravitational field fixes the asymptotic terminal speed that will determine the impact of the superwind in the surroundings, equations (20) and (58).

(iii) The gravitational field can establish different flow regimes and enrichments scenarios that also depend on the steepness and concentration of the galactic parameters: close-box enrichment, equation (18); either nonstationary outflows (outpouring) or open-box enrichment/impoverishment (inpouring), equations (19) and (59); and either accelerating or decelerating stationary outflows, equations (21) and (60). 
(iv) We have established the limits above which selfgravitation and radiative cooling can inhibit the stationary solution. Self-gravitation is likely to be unimportant effect in most cases. Cooling on the other hand affects preferentially to the most massive and compact galaxies with highly concentrated masses and injection rates.

(v) We have evaluated the impact of the gravitational field on the hydrodynamical profiles. We find that the gravitational field can drastically change the expected expansion rate and X-ray emission from the superwind.

(vi) We find that the gravitational field of the extended haloes associated to massive galaxies can drastically alter the free superwind velocity profile and enhance its observable properties. We also find that massive haloes can also contribute to the inhibition of the superwind solution.

(vii) Our model can explain both the observed initial enhancement and posterior flattening of the superwind properties with the galaxy parameters.

(viii) Our model is in agreement with observational data that support the view that metals selectively escape from the least-massive galaxies. However, we demonstrated that, under certain circumstances, a high concentration (i.e. a small concentration parameter $A$ ) can change this.

(ix) In our model, the galaxy total mass $(\mathrm{BM}+\mathrm{DM})$, the mass contained within a bulge or galaxy nucleus (defined by the characteristic radius $r_{\mathrm{sc}}$ ), the mass up to the disc characteristic radius, and the steepness and concentration of the external halo, are all correlated. Since the correlations are nonlinear, deviations from galaxy to galaxy are permitted, see Tables 2 and 3. Oppositely, we assumed no correlation between the above parameters and the concentration and steepness of the mass distribution for $r<r_{\mathrm{sc}}$. This is consistent with the results of the cosmological simulations carried out by Abadi et al. (2010) Navarro et al (2010), in the sense that haloes are not strictly universal. This should be expected, as we based our model in the 'structural contraction' property derived from their simulations. On the other hand, in their extensive work, Salucci et al. (2007) found that the previous parameters were correlated for spiral galaxies, and proposed universal rotation curves assuming a Burkert (1995) profile for the DM distribution. Our theoretical work diverges from theirs in that we considered additionally the mentioned inner concentration and steepness, which traces starburst episodes. Such a consideration discards the possibility of universal halo profiles and rotation curves, since in general this parameters will differ from galaxy to galaxy $\left(r<r_{\mathrm{sc}}\right)$; however, the discrepancy smooths out at larger radii, and thus one could talk of an 'asymptotically universal' property, in the sense defined by Salucci et al. (2007).

\section{ACKNOWLEDGEMENTS}

We thank to the AAS and Timothy Heckman for kindly granting permission for reproducing here fig. 12 from Heckman et al. (2000). We are also grateful to Timothy Heckman for answering our questions about his data.

\section{REFERENCES}

Abadi M. G., Navarro J. F., Fardal M., Babul A., Steinmetz M., 2010, MNRAS, 847
Añorve-Zeferino G. A., 2006, M.Sc. thesis, INAOE

Añorve-Zeferino G. A., Tenorio-Tagle G., Silich S., 2009, MNRAS, 394, 1284

Benson A. J., Madau P., 2003, MNRAS, 344, 835

Blumenthal G. R., Faber S. M., Flores R., Primack J. R., 1986, ApJ, 301, 27

Burkert A., 1995, ApJ, 447, L25

Cairós L. M., Caon N., Papaderos P., Noeske K., Vílchez J. M., García Lorenzo B., Muñoz-Tuñón C., 2003, ApJ, 593, 312

Cantó J., Raga A. C., Rodríguez L. F., 2000, ApJ, 536, 896

Chapman S. C., Blain A. W., Smail I., Ivison R. J., 2005, ApJ, 622,772

Chevalier R. A., Clegg A. W., 1985, Nat, 317, 44

Dahlem M., Weaver K. A., Heckman T. M., 1998, ApJS, 118, 401

Dalcanton J. J., 2007, ApJ, 658, 941

Dawson S., Spinrad H., Stern D., Dey A., van Breugel W., de Vries W., Reuland M., 2002, ApJ, 570, 92

Dehnen W., 1993, MNRAS, 265, 250

Dekel A., Silk J., 1986, ApJ, 303, 39

Dellenbusch K. E., Gallagher J. S., III, Knezek P. M., Noble A. G., 2008, AJ, 135, 326

Ellison S. L., Patton D. R., Simard L., McConnachie A. W., 2008, ApJ, 672, L107

Ellison S. L., Simard L., Cowan N. B., Baldry I. K., Patton D. R., McConnachie A. W., 2009, MNRAS, 396, 1257

Finlator K., Davé R., 2008, MNRAS, 385, 2181

Finlator K., Davé R., 2008, MNRAS, 385, 2181

Frye B., Broadhurst T., Benítez N., 2002, ApJ, 568, 558

Garnett D. R., 2002, ApJ, 581, 1019

Gelover-Santiago A. L., Corona-Galindo M. G., 2007, Rev. Mex. Phys .23, 112

Genzel R., Baker A. J., Tacconi L. J., Lutz D., Cox P., Guilloteau S., Omont A., 2003, ApJ, 584, 633

Governato F., et al., 2010, Nat, 463, 203

Heckman T. M., Armus L., Weaver K. A., Wang J., 1999, ApJ, 517,130

Heckman T. M., Lehnert M. D., Strickland D. K., Armus L., 2000, ApJS, 129, 493

Hernquist L., 1990, ApJ, 356, 359

Hilker M., Baumgardt H., Infante L., Drinkwater M., Evstigneeva E., Gregg M., 2007, A\&A, 463, 119

Jaffe W., 1983, MNRAS, 202, 995

Ji L., Wang Q. D., Kwan J., 2006, MNRAS, 372, 497

Larson R. B., 1974, MNRAS, 169, 229

Lee H., Skillman E. D., Cannon J. M., Jackson D. C., Gehrz R. D., Polomski E. F., Woodward C. E., 2006, ApJ, 647, 970

Lehnert M. D., Heckman T. M., 1995, ApJS, 97, 89

Lehnert M. D., Heckman T. M., 1996, ApJ, 462, 651

Lynden Bell D., 1992, eatc.conf, 270

Mac Low M.-M., Ferrara A., 1999, ApJ, 513, 142

Marlowe A. T., Heckman T. M., Wyse R. F. G., Schommer R., 1995, ApJ, 438, 563

Martin C. L., 2005, ApJ, 621, 227

Martin C. L., Bouché N., 2009, ApJ, 703, 1394

Nath B. B., Trentham N., 1997, MNRAS, 291, 505

Pérez-González P. G., et al., 2005, ApJ, 630, 82

Peeples M. S., Pogge R. W., Stanek K. Z., 2008, ApJ, 685, 904

Peeples M. S., Pogge R. W., Stanek K. Z., 2009, ApJ, 695, 259

Persic M., Salucci P., Stel F., 1996, MNRAS, 281, 27

Pettini M., Shapley A. E., Steidel C. C., Cuby J.-G., Dickinson M., Moorwood A. F. M., Adelberger K. L., Giavalisco M., 2001, ApJ, 554, 981

Rupke D. S., Veilleux S., 2005, ApJ, 631, L37

Rupke D. S., Veilleux S., Sanders D. B., 2005, ApJS, 160, 87

Rupke D. S., Veilleux S., Sanders D. B., 2005, ApJS, 160, 115

Salucci P., Persic M., 1997, ASPC, 117, 1

Sharma M., Nath B.B., 2012, ApJ, 750, 55 
Silich S., Tenorio-Tagle G., Muñoz-Tuñón C., HueyotlZahuantitla F., Wünsch R., Palouš J., 2010, ApJ, 711, 25

Silich S., Tenorio-Tagle G., Rodríguez-González A., 2004, ApJ, 610,226

Stevens I. R., Hartwell J. M., 2003, MNRAS, 339, 280

Strickland D. K., Heckman T. M., 2009, ApJ, 697, 2030

Taniguchi Y., Trentham N., Shioya Y., 1998, ApJ, 504, L79

Tapken C., Appenzeller I., Noll S., Richling S., Heidt J., Meinköhn E., Mehlert D., 2007, A\&A, 467, 63

Tremonti C. A., et al., 2004, ApJ, 613, 898

Turner M. L., et al., 2012, ApJS, 203, 5

Wang B., 1995, ApJ, 444, 590

weaver R., et al., 1977, ApJ, 218, 377

Wünsch R., Silich S., Palouš J., Tenorio-Tagle G., 2007, A\&A, 471,579

Wünsch R., Tenorio-Tagle G., Palouš J., Silich S., 2008, ApJ, 683, 683

\section{APPENDIX A: SELF-GRAVITATION}

For a continuous, non-decelerating solution the density and the sound speed are monotonically decreasing functions of $r$. Thus, self-gravitation becomes important at any radius at which the gas density becomes comparable to the Jeans density. If the superwind is able to escape from a (proto-) galaxy, the density at $r_{\mathrm{sc}}$ has to be less than $\rho_{\mathrm{J}}=c_{\mathrm{sc}}^{2} / G r_{\mathrm{sc}}^{2}$. Using equations (27), (15), and (16) together with the condition $u_{\mathrm{sc}}=c_{\mathrm{sc}}$ we obtain that no solution exists when

$V_{\mathrm{e}} \geq V_{\mathrm{e}, \mathrm{sg}}=\frac{(5-2 \alpha)}{\left(1+\frac{1}{A}\right)}\left[1-\left(\frac{4(2 \eta+1)^{3} G^{2} \dot{E}_{\mathrm{eff}}^{2}}{(4 \pi)^{2} V_{\infty}^{10}}\right)^{1 / 3}\right]$.

The expression between square brackets further reduces the permitted values of $V_{\mathrm{e}}$. An abrupt cut-off occurs when the quantity between parenthesis inside squared brackets becomes unity. We can rewrite this quantity as $\left[R^{4} / u_{\mathrm{sc}}^{4}\right]\left(G^{2} \rho_{s c}^{2}\right)=t_{\mathrm{dyn}}^{4} / t_{\mathrm{ff}}^{4}$, i.e. as the fourth power of the ratio of the dynamical time to the free-fall time. Hence, it recovers the natural time-scales to evaluate self-gravitation effects. When $V_{\mathrm{e}}$ satisfies inequality (A1), both the gravitational field associated to the dynamical mass and selfgravitation inhibit the solution. When $t_{\mathrm{dyn}}^{4} / t_{\mathrm{ff}}^{4}=1$, the responsible is pure self-gravitation. The latter, however, would require extraordinary large energy injection rates and/or mass-loading, see equation (3). Hence, self-gravitation will be an unimportant effect in most cases.

However, for some of the Dehnen-like distributions, selfgravitation (and radiative cooling) can inhibit the stationary flow for $r \ll r_{\mathrm{sc}}$. This is always the case for the central regions of (proto-) galaxies with density distributions steeper than the Herquist-like profile ${ }^{11}(\alpha>1)$, regardless of their other parameters. This is a consequence of the conservation of mass. For such profiles, the injection of mass per unit volume near the object centre is so intense that the resulting gas density would be extremely high (mathematically, infinite). Hence, self-gravitation and cooling will be important there and, as a consequence, the stationary solution will not be continuous in the whole central volume. Instead, it will

11 v.gr. those that follow the Jaffe-like profile, which nevertheless, reproduces the de Vaucouleurs law and can have either an absolute flat or a quasi-flat $v_{\text {rot }}$ curve with a central cusp. adopt bimodal solutions similar to the radiatively induced ones presented by Silich et al. (2010), in which a stationary solution exist only for $r$ larger than a stagnation radius, $r_{\text {st }}$. Here, we will limit ourselves to the case $\alpha \leq 1$ with parameters located below the threshold imposed by the Jeans criteria and our radiative cooling threshold lines, which we present below.

\section{APPENDIX B: RADIATIVE COOLING}

We use a weighed leading order approach to assess the impact of radiative cooling for the case $0 \leq \alpha \leq 1$, i.e. we first expand the velocity in a series of the form

$u \sim \frac{r^{-\alpha}}{\xi} \sum_{k=1}^{\infty} d_{k} r^{k}$

and take just the leading order term, such that $u \sim$ $d_{1} r^{1-\alpha} / \xi$. Here, $\xi$ is the weight factor.

We then integrate equation (4) to obtain

$\rho=\frac{\dot{M}_{\mathrm{eff}}}{4 \pi r_{\mathrm{sc}}^{2}}\left(\frac{1+A}{R+A}\right)^{3-\alpha} \frac{R^{1-\alpha}}{u}$.

Using this equation and the approximation for $u$, we get an equivalent of equation (15) for the radiative case. Written in terms of dimensionless variables, such equation is

$U+2 \eta C=1-\frac{1}{(5-2 \alpha)}\left[(1+A)^{3-\alpha}\left(\frac{R}{R+A}\right)^{2-\alpha} \frac{V_{\mathrm{e}}}{A}\right.$

$\left.-\frac{4 \dot{E} \Lambda \xi^{2} f(A, \alpha, R)}{4 \pi \mu_{\mathrm{n}}^{2} r_{\mathrm{sc}} V_{\infty}^{6} U_{\mathrm{sc}} A}(1+A)^{(3-\alpha)} R^{3-\alpha}\left(\frac{1}{R+A}\right)^{2-\alpha}\right]$

where $U$ and $C$ are $u^{2}$ and $c^{2}$ normalized to $V_{\infty}^{2}, f$ is a function that $\rightarrow(5-2 \alpha) / 3$ as $A \rightarrow \infty$ and blows up at $R=1$ for really small values of $A$ and $\alpha \neq 1$. The second term between square brackets comes from the cooling term: $-\int_{0}^{r} n^{2} \Lambda(T, Z) r^{\prime 2} d r^{\prime} / \rho u r^{2}$. Here, $n=\rho / \mu_{\mathrm{n}}$ is the number density of particles participating in the radiative process, $\mu_{\mathrm{n}}$ is the mean mass per particle for a neutral gas, and $\Lambda(T, Z)$ is the radiative cooling function, which depends on both temperature and metallicity. For a given $Z$, it is not expected to vary more than by a factor of $\sim 2$ in the interval of temperatures of interest $\sim 10^{6}-10^{8} \mathrm{~K}$, and thus, for simplicity, it is taken to be $\Lambda=\Lambda\left(T_{\mathrm{c}}, Z\right)$, where $T_{\mathrm{c}}$ is the temperature ${ }^{12}$ at $r=0$ (Section 3 ). Using the boundary condition at $R=1, U_{\mathrm{sc}}=C_{\mathrm{sc}}$, we obtain a quadratic equation for $U_{\mathrm{sc}}$, with roots:

$U_{\mathrm{sc}}=\frac{1}{2(2 \eta+1)}\left\{\left[1-\frac{V_{\mathrm{e}}\left(1+\frac{1}{A}\right)}{(5-2 \alpha)}\right] \pm\right.$

$\left.\sqrt{\left[1-\frac{V_{\mathrm{e}}\left(1+\frac{1}{A}\right)}{(5-2 \alpha)}\right]^{2}-\frac{16(2 \eta+1) \dot{E} \Lambda \xi^{2} f(1)}{4 \pi \mu_{\mathrm{n}}^{2} r_{\mathrm{sc}} V_{\infty}^{6}} \frac{\left(1+\frac{1}{A}\right)}{(5-2 \alpha)}}\right\}$.

12 Since radiative looses are expected to be larger at the centre of the object. 
From the above formula, we deduce that at least to the leading order, the gravitational inhibition of the flow is uncoupled from and is more decisive (i.e. it is stronger or faster) than the inhibition by radiative cooling, since when the quantity between square brackets vanishes no stationary solution exist at all, independently of the cooling rate. In that case, the flow adopts one of the non-stationary regimes previously described, according to the value of $V_{\mathrm{e}}$. On the other hand, radiative cooling operates only when the gravitational field has not inhibited the solution and inhibits the flow when the quantity under the radical symbol vanishes or becomes negative. From this condition, it is extremely easy to obtain the threshold (maximum) energy deposition rate, $\dot{E}_{\mathrm{th}}$, for which cooling inhibits the stationary solution in terms of $V_{\infty}, r_{\mathrm{sc}}, \alpha, A$ and $V_{\mathrm{e}}$. The last three parameters are the new ingredients incorporated in our formulation. When $V_{\mathrm{e}}=0, \alpha=0$ and $A \rightarrow \infty$ we recover the radiative threshold lines for the standard CC85 model estimated first numerically by Silich, Tenorio-Tagle \& Rodríguez-González (2004), given analytically by Añorve-Zeferino (2006) using a necessary and sufficient condition for the existence of the stationary flow, and generalized later semi-analytically by Wünsch et al. (2007) for CC85-like bimodal outflows. We are also able to reproduce as a particular case the threshold lines numerically obtained by Silich et al. (2010) for a uniform distribution including the gravitational field $\left(V_{\mathrm{e}} \neq 0\right.$, $\alpha=0$ and $A \rightarrow \infty)$.

Equation (B4) contains additional useful information. The physically meaningful root corresponds to the ' + ' sign. This tells us that the radiative losses per unit volume cannot be more than $100(1-1 / \sqrt{2}) \% \sim 29 \%$ of the effective energy injection rate minus the rate at which work is done against the gravitational field, per unit volume. This is in outstanding agreement with previous numerical estimates for the uniform distribution without gravitational field (Silich et al. 2004; Wünsch et al. 2008; see, also, Strickland \& Heckman 2009), and thus, it also confirms the validity of our leading order approach. This also indicates that as in the previous numerical studies, when the stationary solution exist, cooling is not going to modify significantly ${ }^{13}$ the hydrodynamical profiles established by equations (4)-(6).

In astrophysical units, the expression for $\dot{E}_{\mathrm{th}}$ is

$\dot{E}_{\mathrm{th}}=\left(1.1 \times 10^{45} \mathrm{erg} \mathrm{s}^{-1}\right) \frac{(5-2 \alpha) r_{\mathrm{sc}, \mathrm{pc}} V_{8}^{6}\left[1-\frac{V_{\mathrm{e}}\left(1+\frac{1}{A}\right)}{(5-2 \alpha)}\right]^{2}}{(2 \eta+1)(1+1 / A) \xi^{2} f_{1} \Lambda_{1}}$,

where the radius is expressed in parsecs, the effective terminal speed in units of $1000 \mathrm{~km} \mathrm{~s}^{-1}$ and $\Lambda_{1}$ is $\Lambda\left(T_{\mathrm{c}}, Z\right)$ normalized to $10^{-23} \mathrm{erg} \mathrm{s}^{-1} \mathrm{~cm}^{3}$.

The function $f_{1}=f(A, \alpha, 1)$ is given by

$f_{1}=\frac{(1+A)^{-2 \alpha}}{A^{2}(\alpha-2)(2 \alpha-3)}\left\{A^{2 \alpha}(1+A)^{5}-\right.$

13 N.B. In the purely radiative case, when cooling exceeds $29 \%$, it breaks up the stationary solution. In general, it is much less than that

$$
\left.A^{3}(1+A)^{2 \alpha}\left[A^{2}+A(5-2 \alpha)+(\alpha-2)(2 \alpha-5)\right]\right\} .
$$

Although cumbersome, the last function is needed to properly locate the radiative threshold lines in the parameter space ( $f$ came from direct integration). Nevertheless, a useful property is that $f(A, 1,1)=1$, i.e. when evaluated at $R=1, f$ is independent of $A$ when $\alpha=1$. Other values of interest are $f(0.5,0,1)=4$ and $f(0.1,0,1)=80 / 3$. This interest rest on that we obtain asymptotically flat rotation curves for $\alpha \in[0,1]$ when $A \sim 0.1-0.5$.

In turn, the weight factor is given by $\xi^{2}=b^{2} \xi_{0}^{2}$, with

$b=(2 \eta+1)^{1 / 2}\left[\frac{b_{0}}{(2 \eta+1)}\right]^{\frac{b_{0}}{2[\eta(1-\alpha)+(5-\alpha)(\eta+1)]}}$,

$b_{0}=(1-\alpha)(3 \eta+1)+(5-\alpha)(\eta+1)$

and

$$
\begin{aligned}
\xi_{0}^{2}= & \exp \left(\frac{2 \eta(\alpha+1) \alpha^{2}}{(A+1)^{5-\alpha}}+\frac{A}{A+1}\right) \times \\
& \left(1+\frac{1}{(2 \eta+1) A}\right)^{(1-(2 \eta+1) A)} .
\end{aligned}
$$

The expression for $b$ recovers the exact value of the slope for velocity when $r \rightarrow 0$ and $A \rightarrow \infty$, and $\xi_{0}$ is a parametrization that accounts for the error in the approximation $\left(\xi_{0} \rightarrow 1\right.$ when $A \rightarrow \infty$ ).

We will close the current discussion by giving the expression of the galaxy radius for which a flow regime is inhibited by gravity for a given dynamical mass and effective terminal speed:

$r_{\mathrm{sc}, \text { grav }}=\frac{2 G M_{\mathrm{DM}}}{V_{\infty}^{2} V_{\mathrm{e}, \mathrm{th}}}$,

where $V_{\mathrm{e}, \mathrm{th}}$ corresponds to the threshold $V_{\mathrm{e}}$ for one of the possible regimes: accelerating superwind, equation (21); bounded decelerating superwind or possible eventual retention with open-box enrichment, equation (19); or full retention with possible open box enrichment, equation (18). 